\title{
Temperatura crítica de perfis I de aço em situação de incêndio
}

\author{
Critical temperature of I-shaped steel profile in fire \\ situations
}

\section{Valdir Pignatta Silva Arthur Ribeiro Melão}

\section{Resumo}

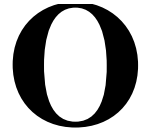

aço e demais materiais estruturais têm sua capacidade resistente reduzida com o aumento de temperatura em situação de incêndio. No caso dos perfis de aço sob campo uniforme de temperaturas, define-se temperatura crítica aquela que os conduz ao colapso. O objetivo deste trabalho foi desenvolver uma ferramenta, que permite determinar de forma expedita a temperatura crítica de pilares e vigas. $\mathrm{O}$ método empregado foi considerar que o colapso ocorrerá quando os valores de cálculo dos esforços solicitantes se igualarem aos esforços resistentes, ambos em situação de incêndio, com base no método simplificado da norma brasileira e aplicando o programa AcoInc, desenvolvido pelos autores. O principal resultado foi uma ferramenta gráfica cuja similararidade não foi encontrada na literatura pesquisada. Seu uso simplificará sobremaneira o método de dimensionamento normatizado. Uma conclusão a ser destacada é aquela em que o valor de temperatura crítica, usualmente admitido na prática $\left(550{ }^{\circ} \mathrm{C}\right)$, podem estar contra a segurança.

Palavras-chaves: Incêndio. Estruturas de aço. Temperatura crítica. Pilar. Viga.

Valdir Pignatta Silva Universidade de São Paulo São Paulo - SP - Brasil

Arthur Ribeiro Melão Universidade de São Paulo São Paulo - SP - Brasil

Recebido em 18/11/16 Aceito em 20/06/17

\section{Abstract}

The resistance of steel and other structural materials is reduced by increased temperatures in a fire situation. In the case of steel profiles under uniform temperatures, the critical temperature is defined as that which leads to collapse. The objective of this study was develop a tool to allow speedy determination of the critical temperature of columns and beams. The method used was based on the simplified procedure of the Brazilian standard and using the AcoInc software developed by the authors. The main result was the graphic tool whose similarity werenot found in the literature. The use of the tool developed in this study simplifies the use of the standard design method. One conclusion to be highlighted is one in which the critical temperature value, usually admitted in practice $\left(550^{\circ} \mathrm{C}\right)$, may be unsafety.

Keywords: Fire. Steel structure. Critical temperature. Column. Beam. 


\section{Introdução}

Os materiais estruturais perdem resistência e módulo de elasticidade com o aumento de temperatura em um incêndio.

A legislação/normatização brasileira requer que todas as edificações tenham a estrutura verificada para a situação de incêndio. É amplamente conhecido que o meio técnico ainda não está familiarizado com tal verificação. Neste trabalho, é apresentada uma ferramenta de cálculo, inédita, visando simplificar essa verificação.

No caso dos elementos de estruturas de aço, vigas e pilares, sujeitos à temperatura uniforme em seu volume, há uma temperatura que os leva ao colapso. Essa é chamada de temperatura crítica.

O objetivo deste trabalho é apresentar uma ferramenta expedita para a determinação da temperatura crítica de pilares e vigas com seção transversal em forma de "I" para os tipos de aço mais usados correntemente.

Silva (2004) construiu curvas que permitem determinar a temperatura crítica de pilares. Essas curvas foram construídas com base nas normas NBR 8800 (ABNT, 1988) e NBR 14323 (ABNT, 1999). Velarde e Silva (2009) atualizou o mesmo estudo, considerando o projeto de revisão das normas brasileiras. Atualmente, ambas as normas já possuem revisões publicadas, NBR 8800 (ABNT, 2008) e NBR 14323 (ABNT, 2013). O presente trabalho atualiza e amplia esses estudos, por exemplo, incluindo a temperatura crítica de vigas de aço sujeitas à instabilidade lateral com torção.

Em Garlock e Kodur (2016), Wang et al. (2013), Vila Real e Franssen (2010), Franssen, Zaharia e Kodur (2009), Parkinson e Kodur (2008), Franssen e Zaharia (2005), Skowronsky (2004), Vila Real (2003), Wang (2002) e demais títulos mencionados neste artigo, não se encontrou nada que se assemelhe aos resultados aqui apresentados, cuja finalidade é agilizar o dimensionamento de estruturas de aço em situação de incêndio.

\section{Método}

\section{Considerações gerais}

Neste trabalho, considerou-se que o colapso é atingido quando o esforço resistente de cálculo se iguala ao esforço solicitante de cálculo, ambos para a situação de incêndio, ou seja, quando o elemento estrutural alcançou o estado limite último, conforme definido em norma.

Portanto, a temperatura crítica de uma barra, seja pilar ou viga, pode ser determinada ao se igualar os esforços solicitantes em situação de incêndio $\left(\mathrm{N}_{\mathrm{fi}, \mathrm{S} d}\right.$ para pilares e $\mathrm{M}_{\mathrm{fi}, S d}$ para vigas) aos resistentes ( $\mathrm{N}_{\mathrm{fi}, \mathrm{Rd}}$ para pilares e $\mathrm{M}_{\mathrm{fi}, \mathrm{Rd}}$ para vigas) na mesma situação. A norma brasileira NBR 14323 (ABNT, 2013) fornece o formulário para a determinação dos esforços resistentes de cálculo em situação de incêndio, enquanto a NBR 8800 (ABNT, 2008) fornece os equivalentes à temperatura ambiente. A NBR 8681 (ABNT, 2003) e a NBR 14323 (ABNT, 2013) indicam a maneira de se determinar a ação de cálculo na situação excepcional do incêndio e, por consequência, os esforços solicitantes de cálculo em incêndio. No item seguinte detalha-se o procedimento adotado.

\section{Procedimento adotado}

\section{Barras comprimidas}

Para perfis I duplamente simétricos, na ausência de instabilidade local e à temperatura ambiente, conforme NBR 8800 (ABNT, 2008), vale a Equação 1.

$\mathrm{N}_{\mathrm{Rd}}=\frac{\chi \mathrm{Af}_{\mathrm{y}}}{1,1}$

Eq. 1

Onde:

$\mathrm{N}_{\mathrm{Rd}}$ é o valor de cálculo da força normal resistente à compressão;

A é a área da seção transversal do perfil;

$\mathrm{f}_{\mathrm{y}}$ é a resistência ao escoamento do aço; e

$\chi$ é o fator redutor da capacidade resistente da barra comprimida, função da imperfeição inicial, não linearidade geométrica e do material (SILVA, 2016), determinado conforme a Equação 2.

para $\lambda_{0} \leq 1,5 \rightarrow \chi=0,658^{\lambda_{0}^{2}}$

para $\lambda_{0}>1,5 \rightarrow \chi=\frac{0,877}{\lambda_{0}^{2}}$

Na Equação 2, $\lambda_{0}$ é a esbeltez reduzida da barra comprimida, que no caso geral é determinada conforme a Equação 3.

$\lambda_{0}=\sqrt{\frac{\mathrm{A} \cdot \mathrm{f}_{\mathrm{y}}}{\mathrm{N}_{\mathrm{e}}}}$

Na Equação 3, $\mathrm{N}_{\mathrm{e}}$ é a menor força crítica entre as calculadas para flambagens por flexão, torção e flexotorção. Entretanto, considerando apenas o caso de instabilidade por flexão, que é o que se verifica nos perfis duplamente simétricos com seção transversal em forma de "I", ou seja, $\mathrm{N}_{\mathrm{e}}=$ 
$\left(\pi^{2} \mathrm{E} \mathrm{I}\right) / \mathrm{L}^{2}$, a Equação 3 transforma-se na Equação 4 .

$$
\lambda_{0}=\frac{\lambda}{\sqrt{\frac{\pi^{2} E}{f_{y}}}}
$$

Eq. 4

Onde:

$\lambda$ é a esbeltez do perfil;

E é o módulo de elasticidade do aço; e

$\mathrm{f}_{\mathrm{y}}$ é a resistência ao escoamento do aço.

Para perfis I duplamente simétricos e na ausência de instabilidade local, segundo a NBR 14323 (ABNT, 2013), em situação de incêndio vale a Equação 5.

$$
\mathrm{N}_{\mathrm{fi}, \mathrm{Rd}}=\chi_{\mathrm{fi}} \mathrm{k}_{\mathrm{y}, \theta} \mathrm{A} \mathrm{f}_{\mathrm{y}}
$$

Onde:

$\mathrm{N}_{\text {fi,Rd }}$ é o valor de cálculo da força normal resistente à compressão em situação de incêndio;

$\mathrm{k}_{\mathrm{y} \theta}$ é o fator de redução da resistência ao escoamento $\left(f_{y}\right)$ do aço em função da temperatura, conforme a Figura 1 e a Tabela 1; e

$\chi_{\text {fi }}$ é o fator redutor da capacidade resistente da barra comprimida em situação de incêndio, determinado conforme a Equação 6.

$$
\chi_{\mathrm{fi}}=\frac{1}{\varphi_{0, \mathrm{fi}}+\sqrt{\varphi_{0, \mathrm{fi}}^{2}-\lambda_{0, \mathrm{fi}}^{2}}}
$$

Na Equação 6:

$$
\begin{aligned}
& \varphi_{0, \mathrm{fi}}=0,5\left(1+\alpha \lambda_{0, \mathrm{fi}}+\lambda_{0, \mathrm{fi}}^{2}\right) ; \\
& \alpha=0,022 \sqrt{\frac{\mathrm{E}}{\mathrm{f}_{\mathrm{y}}}} ;
\end{aligned}
$$

$\lambda_{\text {o,fi }}$ é o índice de esbeltez reduzido em situação de incêndio determinado conforme a Equação 7; e

E é o módulo de elasticidade do aço, valendo $20.000 \mathrm{kN} / \mathrm{cm}^{2}$.

$\lambda_{0 \mathrm{fi}}=\lambda_{0} \sqrt{\frac{k_{y \theta}}{k_{E \theta}}}$

Eq. 7

Na Equação 7, $k_{\mathrm{y}, \theta}$ e $\mathrm{k}_{\mathrm{E} \theta}$ são, respectivamente, os fatores de redução da resistência ao escoamento $\left(\mathrm{f}_{\mathrm{y}}\right)$ e do módulo de elasticidade (E) do aço a altas temperaturas, determinados conforme a Tabela 1 e mais bem visualizados na Figura 1.

Das Equações 1 e 5, tem-se a Equação 8.

$\frac{\mathrm{N}_{\mathrm{fi}, \mathrm{Rd}}}{\mathrm{N}_{\mathrm{Rd}}}=1,1 \frac{\chi_{\mathrm{fi}} \mathrm{k}_{\mathrm{y}, \theta}}{\chi}$

Eq. 8

No instante do colapso, conforme definido no item "Procedimento adotado", $\mathrm{N}_{\mathrm{fi}, \mathrm{Rd}}=\mathrm{N}_{\mathrm{fi}, \mathrm{Sd}}$, em que $\mathrm{N}_{\mathrm{fi}, \mathrm{Sd}}$ é o valor de cálculo da força normal de compressão em situação de incêndio determinada por meio das normas NBR 8681 (ABNT, 2003) ou NBR 14323 (ABNT, 2013). Assim, tem-se a Equação 9.

Eq. $6 \quad \frac{\mathrm{N}_{\mathrm{fi}, \mathrm{sd}}}{\mathrm{N}_{\mathrm{Rd}}}=1,1 \frac{\chi_{\mathrm{fi}} \mathrm{k}_{\mathrm{y}, \theta}}{\chi}$

Eq. 9

Tabela 1 - Fatores de redução

\begin{tabular}{c|c|c}
\hline $\boldsymbol{\theta}_{\mathbf{a}}\left({ }^{\circ} \mathbf{C}\right)$ & $\mathbf{k}_{\mathbf{y}, \boldsymbol{\theta}}=\mathbf{f}_{\mathbf{y}, \boldsymbol{\theta}} / \mathbf{f}_{\mathbf{y}}$ & $\mathbf{k}_{\mathbf{E}, \boldsymbol{\theta}}=\mathbf{E}_{\boldsymbol{\theta}} / \mathbf{E}$ \\
\hline 20 & 1,000 & 1,000 \\
100 & 1,000 & 1,000 \\
200 & 1,000 & 0,900 \\
300 & 1,000 & 0,800 \\
400 & 1,000 & 0,700 \\
500 & 0,780 & 0,600 \\
600 & 0,470 & 0,310 \\
700 & 0,230 & 0,130 \\
800 & 0,110 & 0,090 \\
900 & 0,060 & 0,0675 \\
1000 & 0,040 & 0,0450 \\
1100 & 0,020 & 0,0225 \\
1200 & 0,000 & 0,000 \\
\hline
\end{tabular}


Figura 1 - Variação dos fatores de redução com a temperatura $\theta$

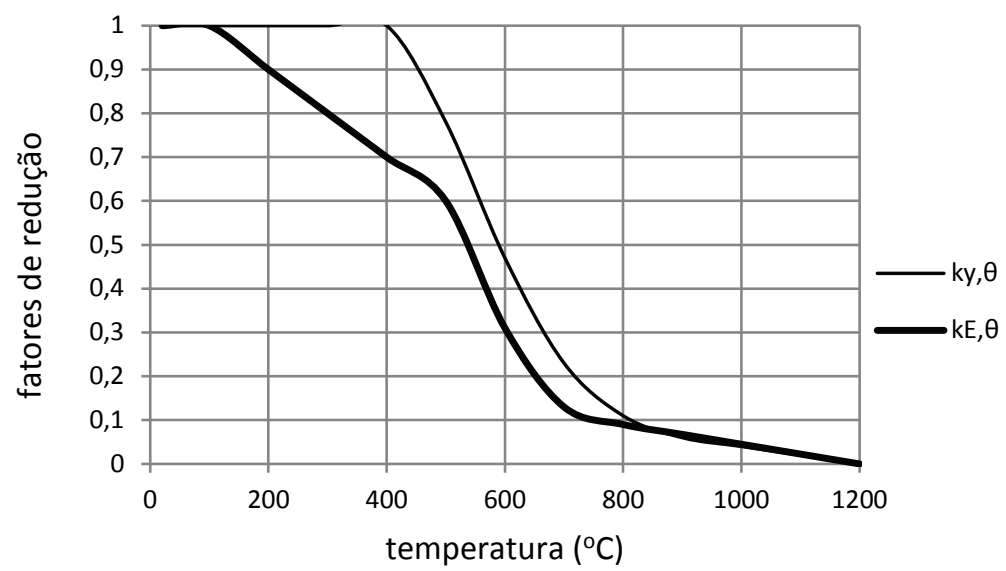

Denominando-se o termo à esquerda da Equação 9 de $\eta$ e explicitando-se as variáveis dessa Equação, têm-se as Equações 10 e 11.

Para $\lambda_{0} \leq 1,5$

$$
\begin{aligned}
\eta= & \frac{1,1 k_{y, \theta}}{0,658^{\lambda_{0}{ }^{2}}} \\
& \times\left\{0,5\left(1+0,022 \lambda_{0} \sqrt{\frac{k_{y, \theta}}{k_{E, \theta}}}+\left(\lambda_{0} \sqrt{\frac{k_{y, \theta}}{k_{E, \theta}}}\right)^{2}\right)\right. \\
& +\left[\left(0,5\left(1+0,022 \lambda_{0} \sqrt{\frac{k_{y, \theta}}{k_{E, \theta}}}+\left(\lambda_{0} \sqrt{\frac{k_{y, \theta}}{k_{E, \theta}}}\right)^{2}\right)\right)^{2}\right. \\
& \left.\left.-\left(\lambda_{0} \sqrt{\frac{k_{y, \theta}}{k_{E, \theta}}}\right)^{2}\right]^{-1 / 2}\right\}^{-1}
\end{aligned}
$$

Eq. 10

Para $\lambda_{0}>1,5$

$$
\begin{aligned}
\eta= & \frac{1,1 k_{y, \theta}}{\frac{0,877}{\lambda_{0}{ }^{2}}} \\
& \times\left\{0,5\left(1+0,022 \lambda_{0} \sqrt{\frac{k_{y, \theta}}{k_{E, \theta}}}+\left(\lambda_{0} \sqrt{\frac{k_{y, \theta}}{k_{E, \theta}}}\right)^{2}\right)\right. \\
& +\left[\left(0,5\left(1+0,022 \lambda_{0} \sqrt{\frac{k_{y, \theta}}{k_{E, \theta}}}+\left(\lambda_{0} \sqrt{\frac{k_{y, \theta}}{k_{E, \theta}}}\right)^{2}\right)\right)^{2}\right. \\
& \left.\left.-\left(\lambda_{0} \sqrt{\frac{k_{y, \theta}}{k_{E, \theta}}}\right)^{2}\right]^{1 / 2}\right\}^{-1}
\end{aligned}
$$

Eq. 11
Observa-se que nas Equações 10a e 10b há uma relação entre $\theta, \eta, \lambda_{0}$ e o material. No instante do colapso, $\theta=\theta_{\mathrm{cr}}$. Quanto ao material, foram escolhidos quatro aços, cujas resistências ao escoamento são: $\mathrm{f}_{\mathrm{y}}=25 \mathrm{kN} / \mathrm{cm}^{2}, \mathrm{f}_{\mathrm{y}}=30 \mathrm{kN} / \mathrm{cm}^{2}, \mathrm{f}_{\mathrm{y}}$ $=34,5 \mathrm{kN} / \mathrm{cm}^{2}$ e $\mathrm{f}_{\mathrm{y}}=35 \mathrm{kN} / \mathrm{cm}^{2}$. Os dois primeiros e o último são mais usados em perfis soldados, e o terceiro adequado aos perfis laminados empregados no Brasil.

Em vista da óbvia dificuldade de obtenção de $\theta_{\mathrm{cr}}$ manualmente, os autores elaboraram um programa de computador, o qual foi inserido no AcoInc. Com esse programa, construíram-se as curvas apresentadas no item "Resultados e comentários". $\mathrm{N}_{\mathrm{Rd}}$ e $\lambda_{0}$ já são conhecidos, decorrentes do dimensionamento à temperatura ambiente. Daí, pelo método gráfico proposto, calcula-se a $\theta_{\text {cr }}$ do pilar.

\section{Barras sob flexão}

Diferente da força normal de compressão, o momento fletor resistente à temperatura ambiente não é tratado pela NBR 8800 (ABNT, 2008) por meio de uma curva $\chi \times \lambda_{o}$, e sim $\mathrm{M}_{\mathrm{Rk}} \times \lambda_{\mathrm{b}}$, conforme a Equação 11, na qual por simplificação e a favor da segurança, adotou-se $C_{b}$ igual a 1 , sendo $C_{b} 0$ fator de modificação para diagrama de momento fletor não uniforme, conforme a NBR 8800 (ABNT, 2008).

$$
\begin{aligned}
M_{\mathrm{Rk}}= & \mathrm{M}_{\mathrm{p}} \text {, para } \lambda_{\mathrm{b}} \leq \lambda_{\mathrm{p}} \\
\mathrm{M}_{\mathrm{Rk}}= & {\left[\mathrm{M}_{\mathrm{p}}-\left(\mathrm{M}_{\mathrm{p}}-\mathrm{M}_{\mathrm{r}}\right) \frac{\lambda-\lambda_{\mathrm{p}}}{\lambda_{\mathrm{r}}-\lambda_{\mathrm{p}}}\right], } \\
& \text { para } \lambda_{\mathrm{p}}<\lambda_{\mathrm{b}} \leq \lambda_{\mathrm{r}}
\end{aligned}
$$

Eq. 12

$\mathrm{M}_{\mathrm{Rk}}=\mathrm{M}_{\mathrm{e}} \leq \mathrm{M}_{\mathrm{p}}$, para $\lambda_{\mathrm{b}}>\lambda_{\mathrm{r}}$ 
Na Equação 12:

$\mathrm{M}_{\mathrm{Rk}}$ é o valor característico do momento resistente da viga;

$\mathrm{M}_{\mathrm{p}}$ é o valor característico do momento de plastificação da seção da viga e igual a $Z_{x} f_{y}$, em que $Z_{x}$ é o módulo resistente plástico em relação ao eixo $\mathrm{x}$, de maior inércia;

$\mathrm{M}_{\mathrm{r}}$ é o momento fletor limite entre o regime elástico linear e o elastoplástico e igual a $0,7 \mathrm{f}_{\mathrm{y}} \mathrm{W}_{\mathrm{x}}$, em que $\mathrm{W}_{\mathrm{x}}$ é o módulo resistente elástico em relação ao eixo x, de maior inércia;

$\mathrm{M}_{\mathrm{e}}$ é o momento crítico de flambagem elástica fornecido pela NBR 8800 (ABNT, 2008);

$\lambda_{\mathrm{b}}$ é a esbeltez associada ao $\lambda_{\mathrm{b}}$, que é a distância entre travamentos laterais;

$\lambda_{\mathrm{p}}$ é a esbeltez que determina o final do regime plástico e igual a $1,76 \sqrt{\frac{E}{f_{y}}}$;

$\lambda_{\mathrm{r}}$ é a esbeltez limite entre o regime elástico linear do elastoplástico e pode ser encontrado em tabelas, por exemplo, em Silva (2012).

Dessa forma, os autores fizeram uma alteração de variáveis para chegar à relação $\chi_{\text {FLT }} \times \lambda_{0, F L T}$ adimensional, conforme as Equações 13 e 14 .

$\chi_{\mathrm{FLT}}=\frac{\mathrm{M}_{\mathrm{Rk}}}{\mathrm{M}_{\mathrm{p}}}$

$\lambda_{0, \mathrm{FLT}}=\sqrt{\frac{\mathrm{M}_{\mathrm{p}}}{\mathrm{M}_{\mathrm{e}}}}$

Empregando-se as Equações 12 a 14 tem-se a Equação 15.

Para $\lambda_{0, \mathrm{FLT}} \leq \sqrt{\frac{\mathrm{M}_{\mathrm{p}}}{\mathrm{M}_{\mathrm{e}, \mathrm{p}}}}$

$\chi_{\mathrm{FLT}}=1$

Para $\lambda_{0, \mathrm{FLT}, \mathrm{p}}<\lambda_{\mathrm{o}, \mathrm{FLT}} \leq \lambda_{0, \mathrm{FLT}, \mathrm{r}}$

$\chi_{\mathrm{FLT}}=1-\left(1-\frac{0,7 \mathrm{~W}_{\mathrm{x}}}{\mathrm{Z}_{\mathrm{X}}}\right) \frac{\lambda_{0, \mathrm{FLT}}-\lambda_{0, \mathrm{FLT}, \mathrm{p}}}{\lambda_{0, \mathrm{FLT}, \mathrm{r}}-\lambda_{0, \mathrm{FLT}, \mathrm{p}}}$

Eq. 15

Para $\lambda_{\mathrm{o}, \mathrm{FLT}} \geq \lambda_{\mathrm{o}, \mathrm{FLT}, \mathrm{r}}=\sqrt{\frac{\mathrm{z}_{\mathrm{x}}}{0,7 \mathrm{~W}_{\mathrm{x}}}}$

$\chi_{\mathrm{FLT}}=\frac{1}{\lambda_{\mathrm{o}, \mathrm{FLT}}^{2}}$

Na Equação 15:

$\lambda_{0, \mathrm{FLT}, \mathrm{p}}=\sqrt{\frac{\mathrm{M}_{\mathrm{p}}}{\mathrm{M}_{\mathrm{e}, \mathrm{p}}}} ; \mathrm{e}$

$\lambda_{0, \mathrm{FLT}, \mathrm{r}}=\sqrt{\frac{\mathrm{M}_{\mathrm{p}}}{\mathrm{M}_{\mathrm{r}}}}=\sqrt{\frac{\mathrm{Z}_{\mathrm{x}}}{0,7 \mathrm{~W}_{\mathrm{x}}}}$.
$\mathrm{M}_{\mathrm{e}, \mathrm{p}}$ é o momento crítico de flambagem elástica para o comprimento limite de plastificação, ou seja, $\lambda_{p} r_{y}$, em que $r_{y}$ é o raio de inércia em torno do eixo de menor inércia, $\mathrm{y}$.

Para perfis I duplamente simétricos e na ausência de instabilidade local, segundo a NBR 14323 (ABNT, 2013), em situação de incêndio vale a Equação 16.

$M_{f i, R d}=\kappa \chi_{F L T, f i} Z_{x} f_{y} \mathrm{k}_{\mathrm{y}, \theta}$

Eq. 16

Na Equação 16:

$\mathrm{M}_{\mathrm{fi}, \mathrm{Rd}}$ é o valor de cálculo momento fletor resistente em situação de incêndio;

$\chi_{\mathrm{FLT}, \text { fi }}$ tem o mesmo formato de $\chi_{\mathrm{fi}}$ (Equação 6), mas alterando o valor de $\lambda_{o, f i}$ (Equação 7), conforme a Equação 17; e

$\kappa$ é um fator de correção que leva em conta o efeito benéfico de uma eventual distribuição de temperatura não uniforme ao longo da altura da seção transversal e vale entre 1,0 e 1,4 conforme a NBR 14323 (ABNT, 2013).

$\lambda_{\mathrm{o}, \mathrm{FLT}, \mathrm{fi}}=\sqrt{\frac{\mathrm{k}_{\mathrm{y}, \theta}}{\mathrm{k}_{\mathrm{y}, \theta}}} \sqrt{\frac{\mathrm{M}_{\mathrm{p}}}{\mathrm{M}_{\mathrm{e}}}}$

Da mesma forma que foi feito para força normal, será criada uma relação adimensional $\mu$, conforme a Equação 18, em que $M_{\mathrm{Rd}}$ é o valor de cálculo momento fletor resistente à temperatura ambiente.

$\mu=\frac{\mathrm{M}_{\mathrm{fi}, \mathrm{Rd}}}{\mathrm{M}_{\mathrm{Rd}}}=1,1 \frac{\kappa k_{y, \theta} \chi_{F L T, f i}}{\chi_{\mathrm{FLT}}}$

Eq. 18

$\mu$ é determinado pela Equação 19.

Para $\lambda_{0, \mathrm{FLT}} \leq \sqrt{\frac{\mathrm{M}_{\mathrm{p}}}{\mathrm{M}_{\mathrm{e}, \mathrm{p}}}}$

$\mu=1,1 \kappa k_{y, \theta}$

$\times\left\{0,5\left(1+0,022 \lambda_{0, F L T} \sqrt{\frac{k_{y, \theta}}{k_{E, \theta}}}+\left(\lambda_{0, F L T} \sqrt{\frac{k_{y, \theta}}{k_{E, \theta}}}\right)^{2}\right)\right.$

$+\left[\left(0,5\left(1+0,022 \lambda_{0, F L T} \sqrt{\frac{k_{y, \theta}}{k_{E, \theta}}}+\left(\lambda_{0, F L T} \sqrt{\frac{k_{y, \theta}}{k_{E, \theta}}}\right)^{2}\right)\right]^{2}\right.$

$\left.-\left(\lambda_{0, F L T} \sqrt{\frac{k_{y, \theta}}{k_{E, \theta}}}\right)^{2}\right\}^{1 / 2}$

Para $\sqrt{\frac{\mathrm{M}_{\mathrm{p}}}{\mathrm{M}_{\mathrm{e}, \mathrm{p}}}}<\lambda_{0, \text { FLT }}<\sqrt{\frac{\mathrm{Z}_{\mathrm{x}}}{0,7 \mathrm{~W}_{\mathrm{x}}}}$ 


$$
\begin{aligned}
\mu= & \kappa k_{y, \theta} \\
& \times\left\{0,5\left(1+0,022 \lambda_{0, F L T} \sqrt{\frac{k_{y, \theta}}{k_{E, \theta}}}+\left(\lambda_{0, F L T} \sqrt{\frac{k_{y, \theta}}{k_{E, \theta}}}\right)^{2}\right)\right. \\
& +\left[\left(0,5\left(1+0,022 \lambda_{0, F L T} \sqrt{\frac{k_{y, \theta}}{k_{E, \theta}}}+\left(\lambda_{0, F L T} \sqrt{\frac{k_{y, \theta}}{k_{E, \theta}}}\right)^{2}\right)\right)^{2}\right. \\
& \left.\left.-\left(\lambda_{0, F L T} \sqrt{\frac{k_{y, \theta}}{k_{E, \theta}}}\right)^{2}\right]^{1 / 2}\right\}^{-1} \\
& \times 1,1\left\{1-\left(1-\frac{0,7 W_{x}}{Z_{x}}\right) \frac{\lambda_{0, F \mathrm{FT}}-\sqrt{\frac{\mathrm{M}_{\mathrm{p}}}{\mathrm{M}_{\mathrm{e}, \mathrm{p}}}}}{\sqrt{\frac{\mathrm{z}_{\mathrm{x}}}{0,7 \mathrm{w}_{\mathrm{x}}}}-\sqrt{\frac{\mathrm{m}_{\mathrm{p}}}{\mathrm{M}_{\mathrm{e}, \mathrm{p}}}}}\right\}^{-1}
\end{aligned}
$$

Eq. 19

$$
\begin{aligned}
& \text { Para } \lambda_{0, \mathrm{FLT}} \geq \sqrt{\frac{\mathrm{Z}_{\mathrm{x}}}{0,7 \mathrm{~W}_{\mathrm{x}}}} \\
& \mu=1,1 k_{y, \theta} \lambda_{0, F L T}{ }^{2} \\
& \times\left\{0,5\left(1+0,022 \lambda_{0, F L T} \sqrt{\frac{k_{y, \theta}}{k_{E, \theta}}}+\left(\lambda_{0, F L T} \sqrt{\frac{k_{y, \theta}}{k_{E, \theta}}}\right)^{2}\right)\right. \\
& +\left[\left(0,5\left(1+0,022 \lambda_{0, F L T} \sqrt{\frac{k_{y, \theta}}{k_{E, \theta}}}+\left(\lambda_{0, F L T} \sqrt{\frac{k_{y, \theta}}{k_{E, \theta}}}\right)^{2}\right)\right)^{2}\right. \\
& \left.-\left(\lambda_{0, F L T} \sqrt{\frac{k_{y, \theta}}{k_{E, \theta}}}\right)^{2}\right\}^{1 / 2}
\end{aligned}
$$

Para a série de perfis laminados da Gerdau, foi calculado o valor de $\sqrt{\frac{\mathrm{M}_{\mathrm{p}}}{\mathrm{M}_{\mathrm{e}, \mathrm{p}}}}$, obtendo-se a Tabela 2 .

Tabela 2 - Valores de $\sqrt{\frac{\mathbf{M}_{\mathbf{p}}}{\mathbf{M}_{\mathbf{e}, \mathbf{p}}}}$

\begin{tabular}{c|c|c}
\hline \multirow{2}{*}{$\mathbf{f}_{\mathbf{y}} \mathbf{( k N / \mathbf { c m } ^ { 2 } )}$} & \multicolumn{2}{|c}{$\sqrt{\frac{\mathbf{M}_{\mathbf{p}}}{\mathbf{M}_{\mathbf{e}, \mathbf{p}}}}$} \\
\hline & Variação & Valor adotado \\
25 & Entre 0,48 e 0,55 & 0,48 \\
30 & Entre 0,52 e 0,60 & 0,52 \\
34,5 & Entre 0,56 e 0,65 & 0,56 \\
35 & Entre 0,56 e 0,65 & 0,56 \\
\hline
\end{tabular}

Entre as planilhas mencionadas, a última é aquela que determina a temperatura crítica de vigas e pilares "I" (Figura 3). Tal planilha constrói gráficos que permitem, a partir de alguns dados, a determinação gráfica expedita da temperatura crítica com base no método simplificado da NBR 14323 (ABNT, 2013). Os dados são incluídos conforme "1" e "2" da Figura 3.

Os aços empregados neste trabalho são aqueles com resistência ao escoamento $\mathrm{f}_{\mathrm{yk}}$ igual a 345 $\mathrm{MPa}$, aço comumente empregado em perfis laminados, e $\mathrm{f}_{\mathrm{yk}}$ iguais a $250 \mathrm{MPa}, 300 \mathrm{MPa}, 345$ MPa e $350 \mathrm{MPa}$, aços usualmente empregados em perfis soldados.

Para fins de temperatura crítica, o AcoInc funciona da seguinte forma:

Arbitram-se valores de $\lambda_{0}$ e $\mu$;

Arbitra-se um valor de $\theta$;

Varia-se $\theta$ até encontrar $\mu$, nesse instante tem-se um ponto no gráfico;

Altera-se $\lambda_{o}$ e depois $\mu$.
Observa-se que na Equação 17 há uma relação entre $\theta, \mu, \lambda_{0}$ e o material. Novamente, lembre-se de que no colapso, $\theta=\theta_{\mathrm{cr}}, \lambda_{0}$ e $\mathrm{M}_{\mathrm{Rd}}$, já são conhecidos do dimensionamento à temperatura ambiente e $\mathrm{M}_{\mathrm{fi}, \mathrm{Sd}}$ é determinável. Os materiais são os mesmos empregados para os pilares.

Da mesma forma que para o pilar, há uma óbvia dificuldade de obtenção de $\theta_{\text {cr }}$ manualmente. Os autores elaboraram um programa de computador, o qual foi também inserido no AcoInc. Com esse programa, construíram-se as curvas apresentadas no item "Resultados e comentários".

$M_{\text {Rd já é conhecido, decorrente do }}$ dimensionamento à temperatura ambiente. $\mathrm{M}_{\mathrm{fi}, \mathrm{Sd}}$ é igual a $\mathrm{M}_{\mathrm{fi}, \mathrm{Rd}}$. Portanto, conhece-se $\mu$, pela Equação 16. $\lambda_{0}$ é determinado pela Equação 12. Daí, pelo método gráfico proposto, calcula-se a $\theta_{\text {cr }}$ da viga.

Para os gráficos, usou-se $\kappa=1$, mas, para outros valores de $\kappa$ eles também podem ser usados com a seguinte estratégia: a partir da $\theta_{\text {cr }}$ determinada com

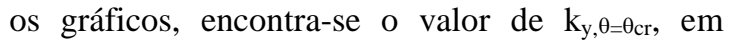


seguida calcula-se a nova $\theta_{\mathrm{cr}}$, aproximadamente, a partir de $\mathrm{k}_{\mathrm{y}, \theta}=\mathrm{k}_{\mathrm{y}, \theta=\theta_{\mathrm{cr}} / \kappa}$.

\section{Programa empregado}

Os autores desenvolveram uma pasta de trabalho, AcoInc, que contém nove planilhas eletrônicas (Figura 2), desenvolvidas em Excel, que incluem várias utilidades para o estudo de perfis I em situação de incêndio (MELÃO, 2016), entre elas a determinação dos esforços resistentes em situação de incêndio e à temperatura ambiente.

Para a criação das planilhas, adotaram-se recursos em Macro, que utilizam linguagem de programação Visual Basic for Applications (VBA) associada ao programa de computador Excel, tornando as planilhas mais ágeis. Com uma programação específica, tornaram-se práticas as execuções de comandos repetitivos que dependem de vários parâmetros para fins de se obter tabelas e gráficos de forma automática. Para facilitar o cálculo em situações, por exemplo, em que o procedimento de cálculo dependa de mais de um fator, foram utilizados os recursos do Visual Basic associado ao Microsoft Excel para criação de funções denominadas pelo programa de “fórmulas". A grande vantagem é de se manter fórmulas que contenham várias condicionais (funções se()) mais compactas no Excel e permitir a visualização das rotinas de cálculo e, portanto, evitar erros e facilitar alterações.

Figura 2 - Planilhas da pasta de trabalho do Acolnc

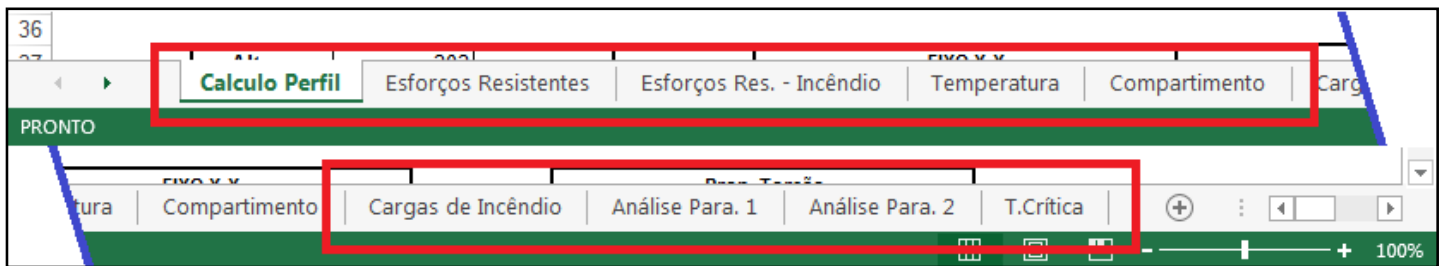

Figura 3 - Planilha para gerar ábacos de temperatura crítica

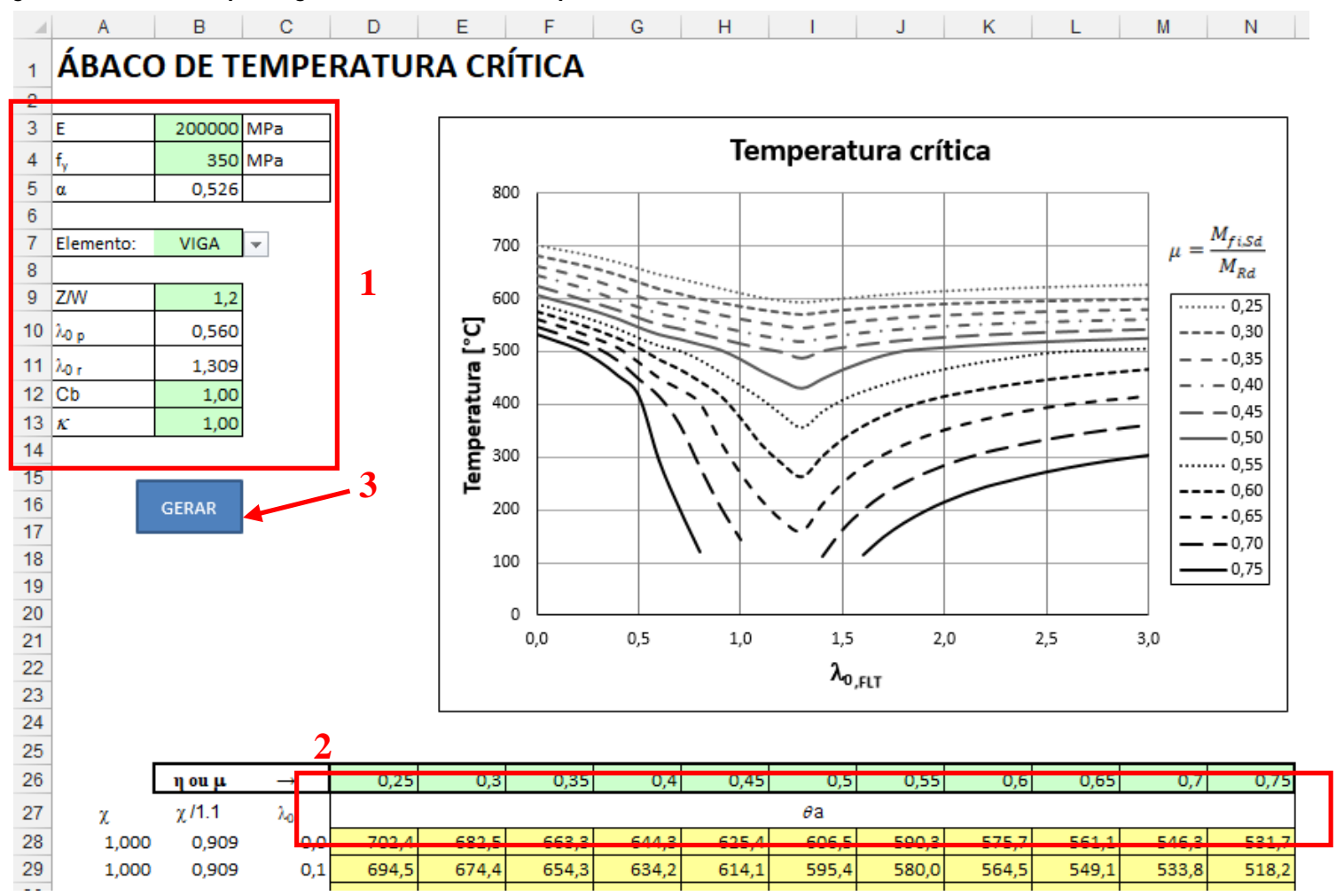

Nota: Legenda:

E é o módulo de elasticidade do aço, à temperatura ambiente;

$f_{y}$ é a resistência ao escoamento do aço, à temperatura ambiente;

a e $\lambda_{0}$ são os parâmetros auxiliares utilizados na formulação de cálculo dos esforços resistentes;

Z e W são os módulos resistentes plástico e elástico, respectivamente; e

$\eta$ e $\mu$ são os níveis de carregamento a serem explicados mais adiante. 


\section{Resultados e comentários}

Na Figura 3 nota-se que se pode escolher o tipo de elemento (viga ou pilar), o tipo de aço $\left(f_{y k}\right)$ e a relação entre os módulos resistentes plástico (Z) e elástico (W). Em seguida, escolhe-se a faixa de variação de $\eta$ (para pilares) ou de $\mu$ (para vigas), que significa a relação entre o esforço solicitante de cálculo em situação de incêndio e o esforço resistente à temperatura ambiente, conforme as Equações 20 e 21.

$\mu=\frac{\mathrm{M}_{\mathrm{fi}, \mathrm{Sd}}}{\mathrm{M}_{\mathrm{Rd}}}$

$\eta=\frac{N_{\text {fi,Sd }}}{N_{R d}}$

Com esses dados de entrada, o programa constrói a curva que permitirá determinar a temperatura crítica a partir do índice de esbeltez reduzido $\left(\lambda_{0}\right)$ à temperatura ambiente.

Tomou-se o cuidado de facilitar ao máximo o uso das curvas por parte do usuário pouco afeito ao dimensionamento em incêndio. Os esforços solicitantes de cálculo em situação de incêndio são fáceis de serem calculados e os esforços resistentes, bem como a esbeltez reduzida, já devem constar do memorial de cálculo da estrutura à temperatura ambiente.

A equação que permite determinar o índice de esbeltez reduzido $\lambda_{0}$ para pilares à temperatura ambiente é fornecida pela NBR 8800 (ABNT, 2008), conforme a Equação 4.

Entretanto, para vigas, foi necessário criar um índice, $\lambda_{0, \mathrm{FLT}}$, especificamente para facilitar este estudo, calculado pela Equação 14.

O AcoInc calcula para cada perfil os valores de $\mathrm{N}_{\mathrm{Rd}}, \mathrm{M}_{\mathrm{Rd}}, \mathrm{N}_{\mathrm{fi}, \mathrm{Rd}}$ e $\mathrm{M}_{\mathrm{fi}, \mathrm{Rd}}$. Adotando um nível de carregamento $\mu$ ou $\eta$, é possível encontrar, em função do índice de esbeltez reduzido, uma temperatura de forma a igualar $\mathrm{N}_{\mathrm{fi}, S d}$ a $\mathrm{N}_{\mathrm{fi}, \mathrm{Rd}}$ (para pilares) ou $\mathrm{M}_{\mathrm{fi}, \mathrm{Sd}}$ a $\mathrm{M}_{\mathrm{fi}, \mathrm{Rd}}$ (para vigas). Essa temperatura é denominada temperatura crítica e pode ser obtida por meio dos gráficos das Figuras 4 a 15. Na construção dos gráficos das Figuras 8 a 15 , por simplificação e a favor da segurança, adotou-se $\mathrm{C}_{\mathrm{b}}$ igual a 1 .

Figura 4 - Temperatura crítica de pilares em função do índice de esbeltez reduzido e do nível de carregamento para $f_{y}=250 \mathrm{MPa}$

\section{Temperatura crítica}

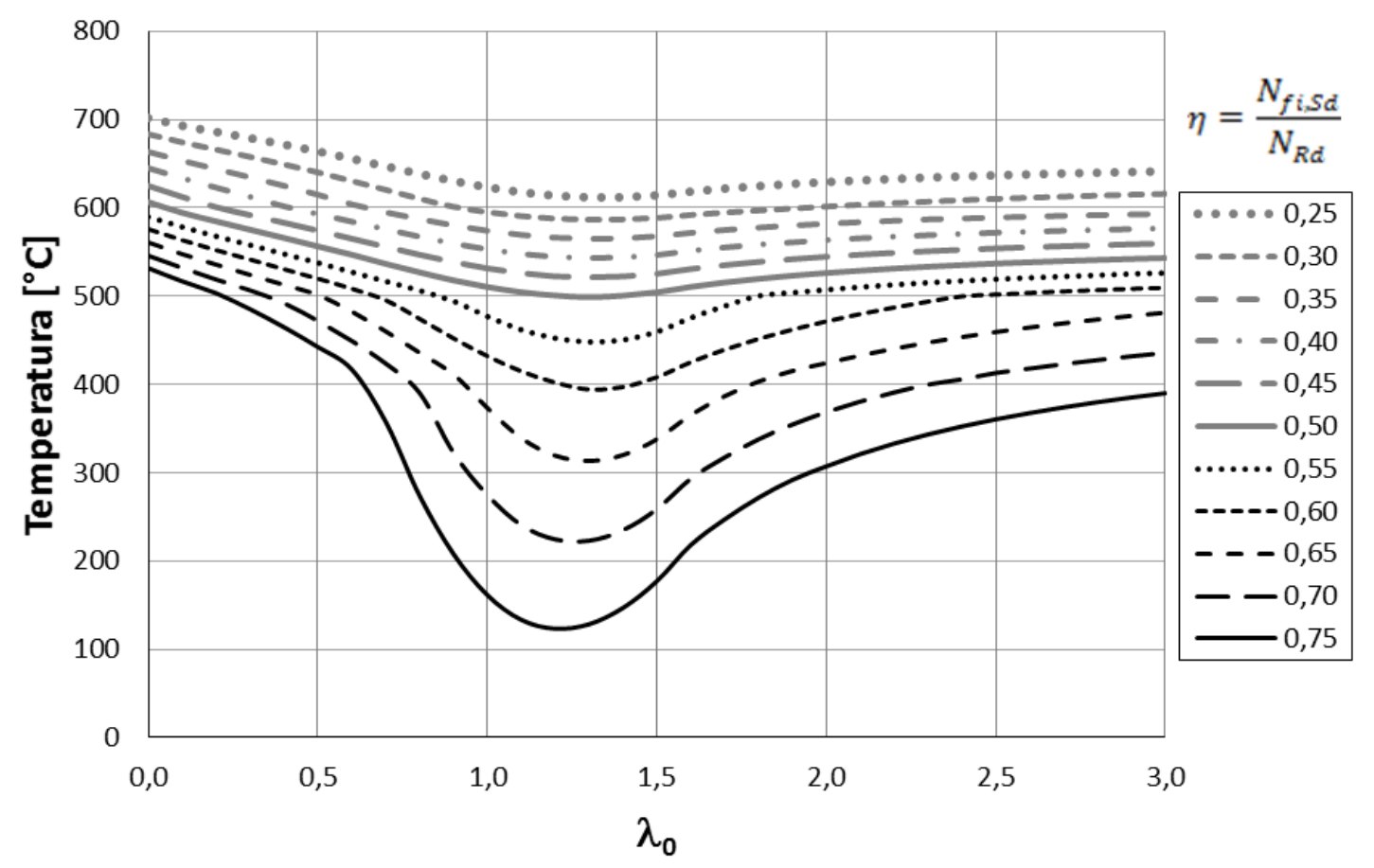

332 Silva, V. P.; Melão, A. R. 
Figura 5 - Temperatura crítica de pilares em função do índice de esbeltez reduzido e do nível de carregamento para $f_{y}=300 \mathrm{MPa}$

\section{Temperatura crítica}

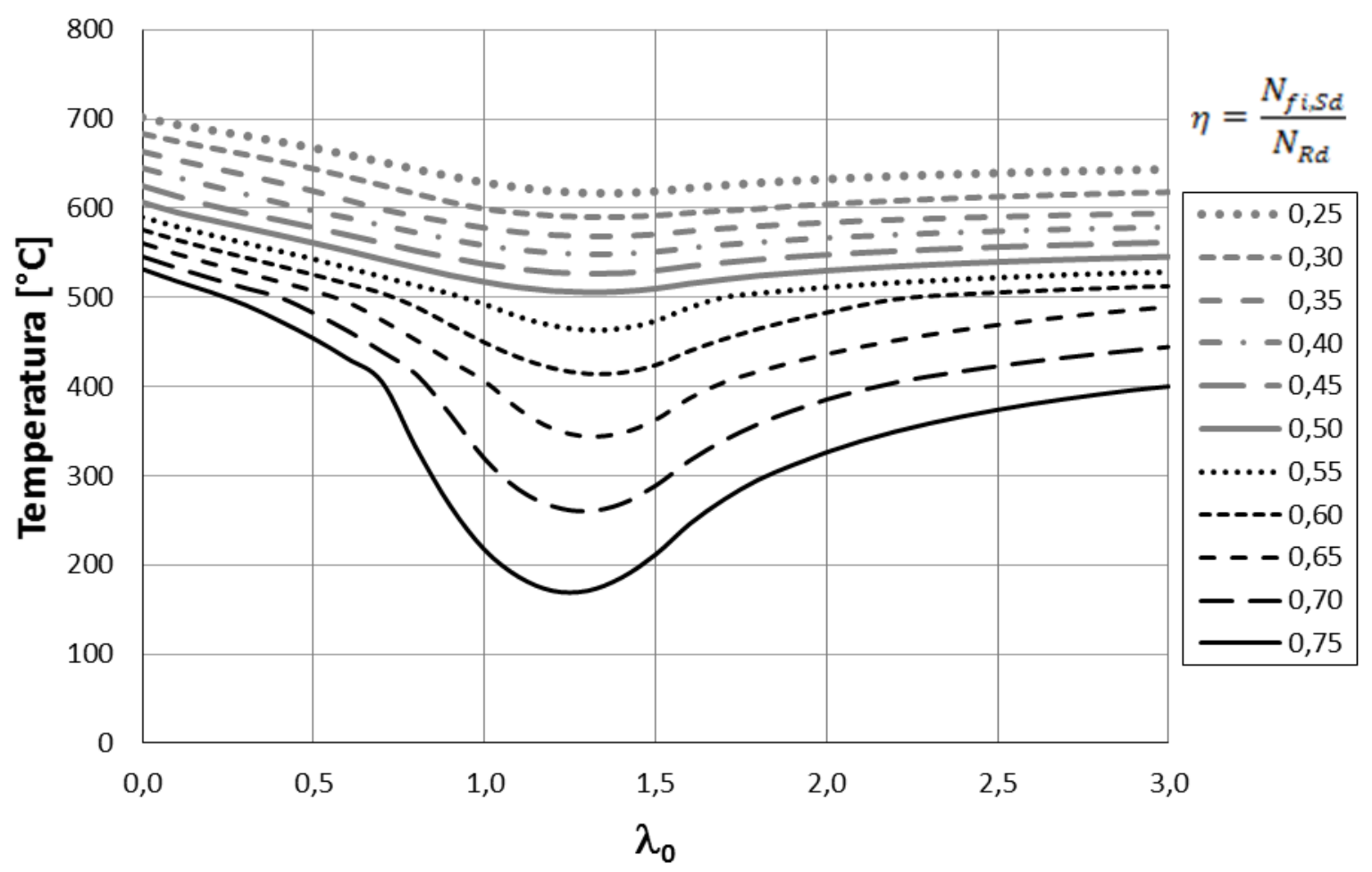

Figura 6 - Temperatura crítica de pilares em função do índice de esbeltez reduzido e do nível de carregamento para $f_{y}=345 \mathrm{MPa}$

\section{Temperatura crítica}

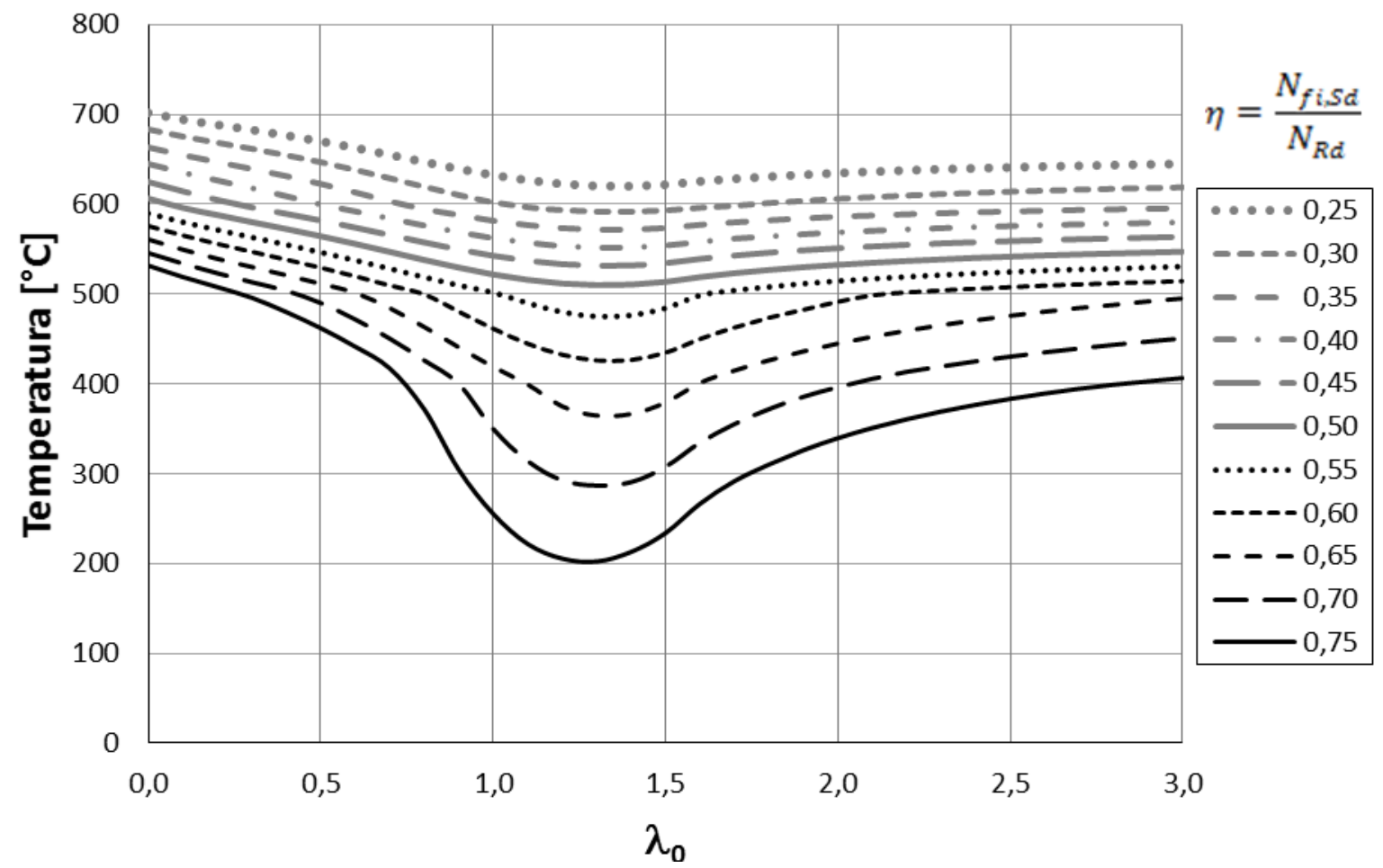


Figura 7 - Temperatura crítica de pilares em função do índice de esbeltez reduzido e do nível de carregamento para $f_{y}=350 \mathrm{MPa}$

\section{Temperatura crítica}

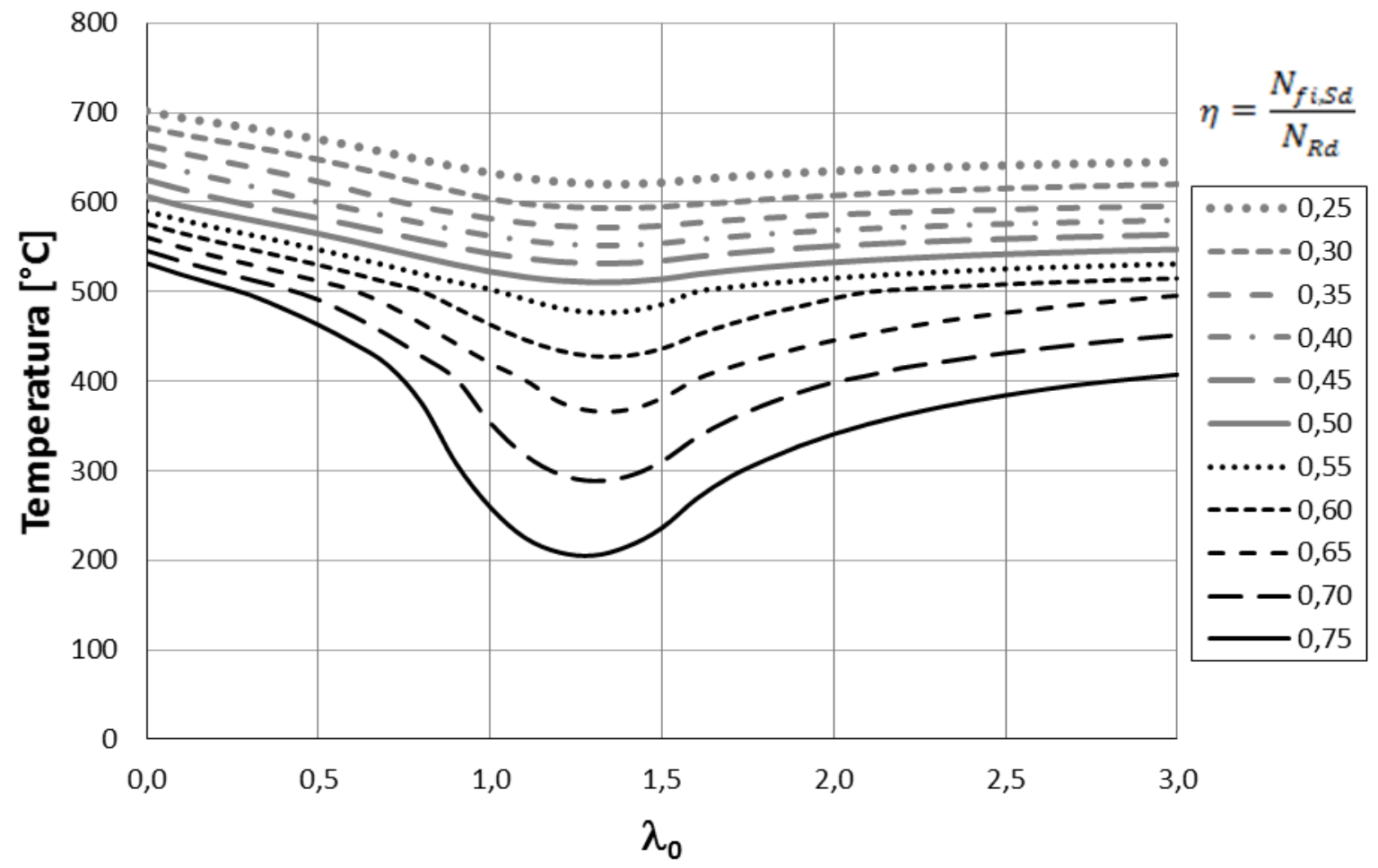

Figura 8 - Temperatura crítica de vigas em função do índice de esbeltez reduzido e do nível de carregamento, para $f_{y}=250 \mathrm{MPa}$ e $\mathrm{Z} / \mathrm{W}=\mathbf{1}, \mathbf{1 0}$

\section{Temperatura crítica}

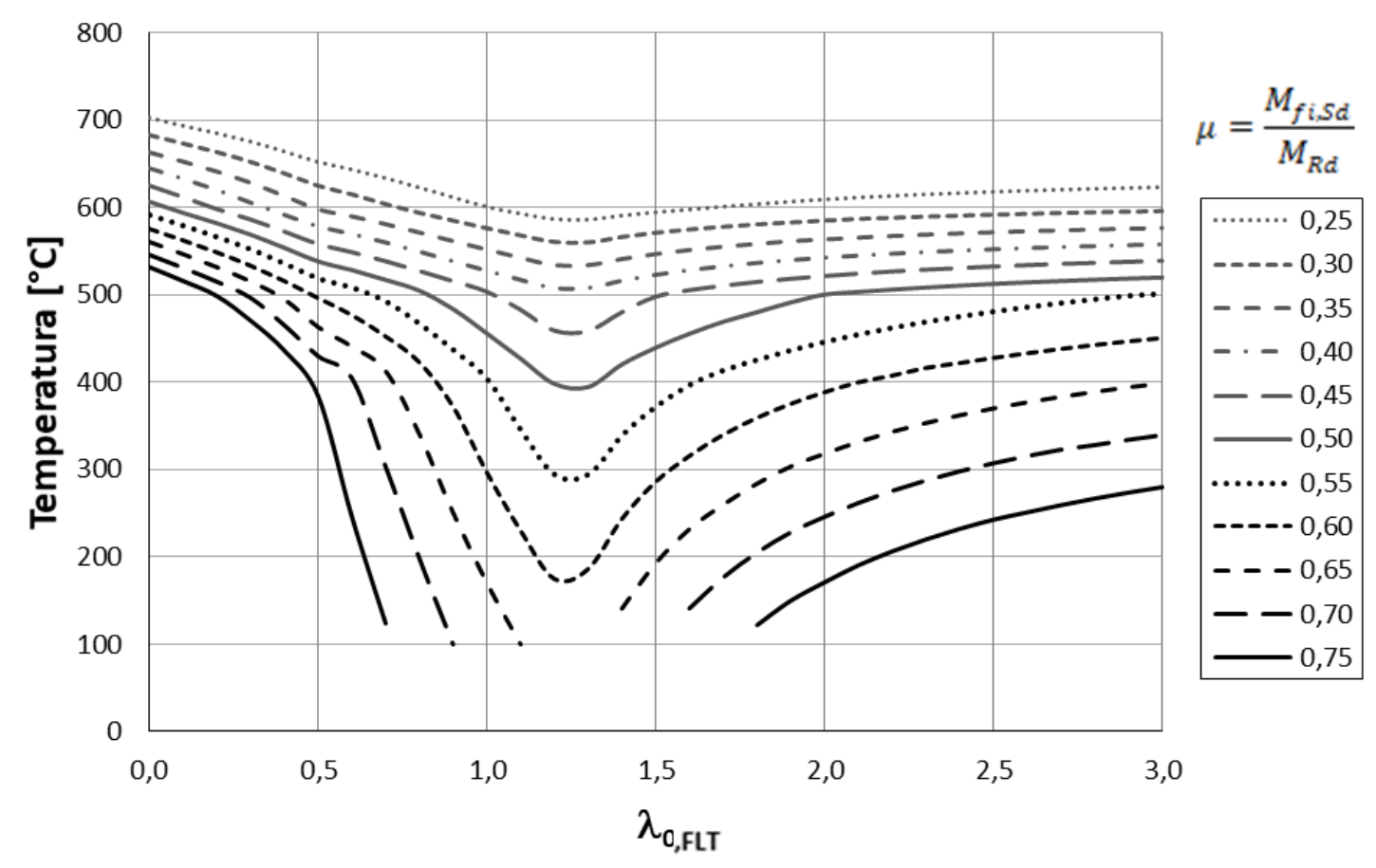


Figura 9 - Temperatura crítica de vigas em função do índice de esbeltez reduzido e do nível de carregamento, para $\mathrm{f}_{\mathrm{y}}=300 \mathrm{MPa}$ e $\mathrm{Z} / \mathrm{W}=1,10$

\section{Temperatura crítica}

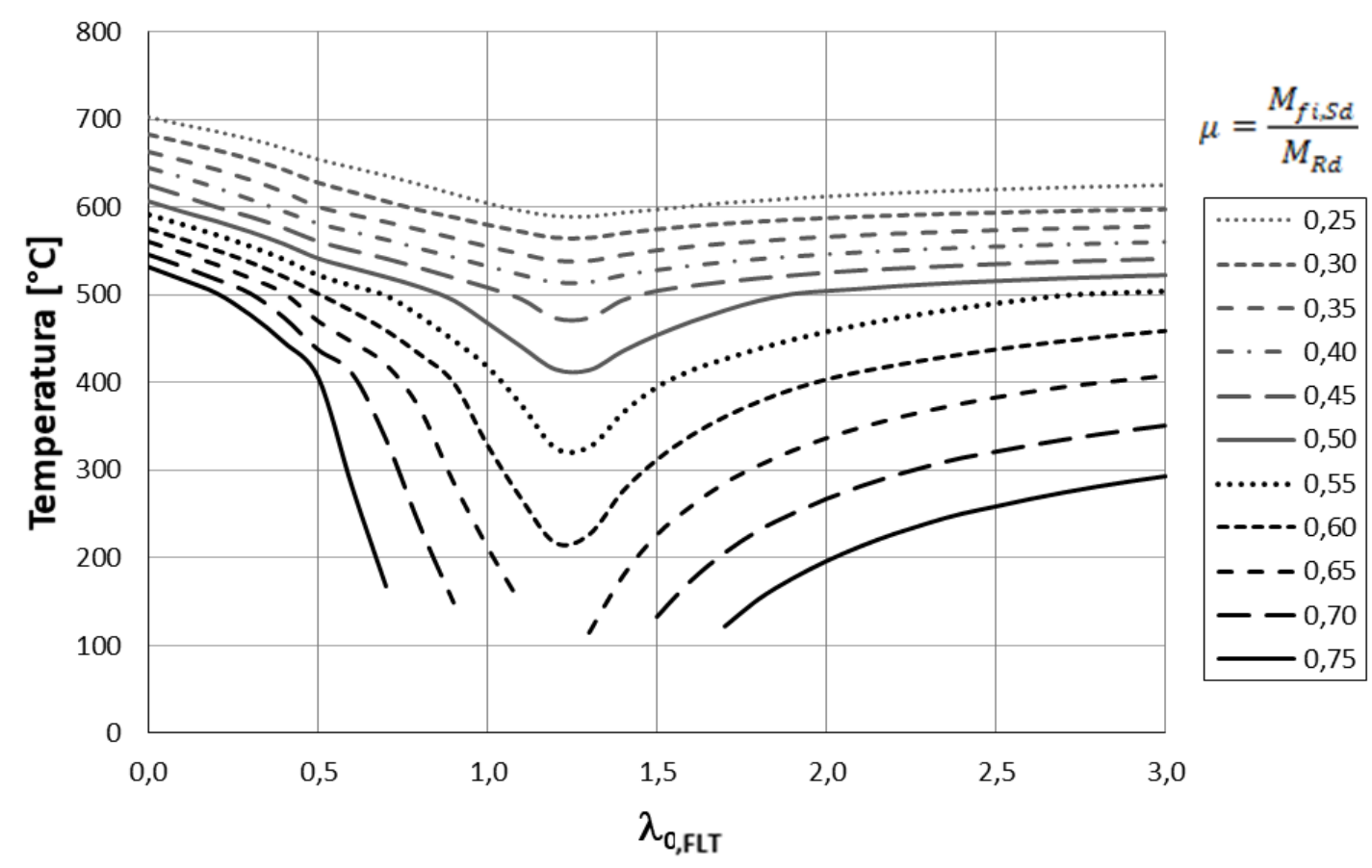

Figura 10 - Temperatura crítica de vigas em função do índice de esbeltez reduzido e do nível de carregamento, para $\mathrm{f}_{\mathrm{y}}=345 \mathrm{MPa}$ e $\mathrm{Z} / \mathrm{W}=1,10$

\section{Temperatura crítica}

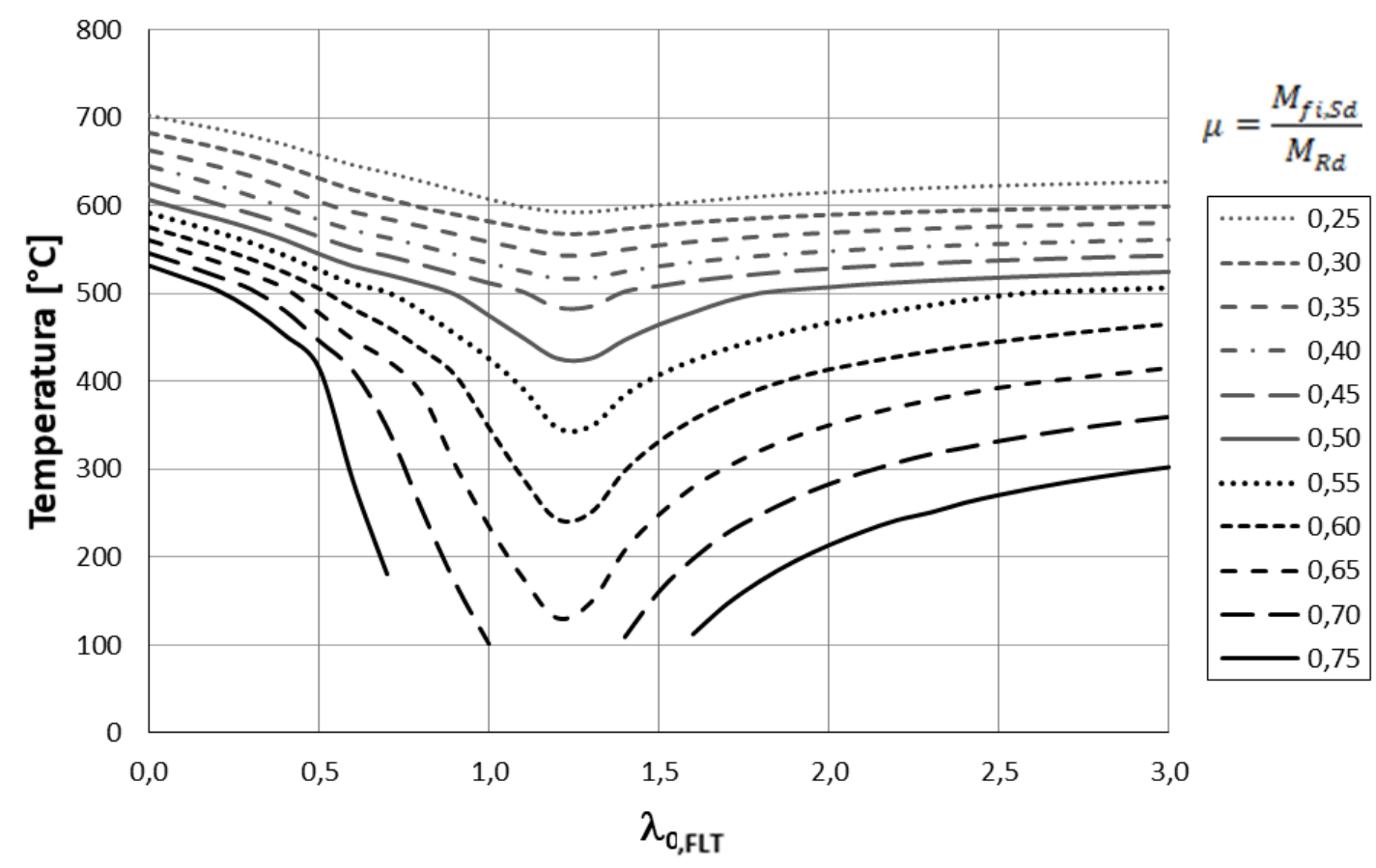


Figura 11 - Temperatura crítica de vigas em função do índice de esbeltez reduzido e do nível de carregamento, para $f_{y}=350 \mathrm{MPa}$ e $\mathrm{Z} / \mathrm{W}=1,10$

\section{Temperatura crítica}

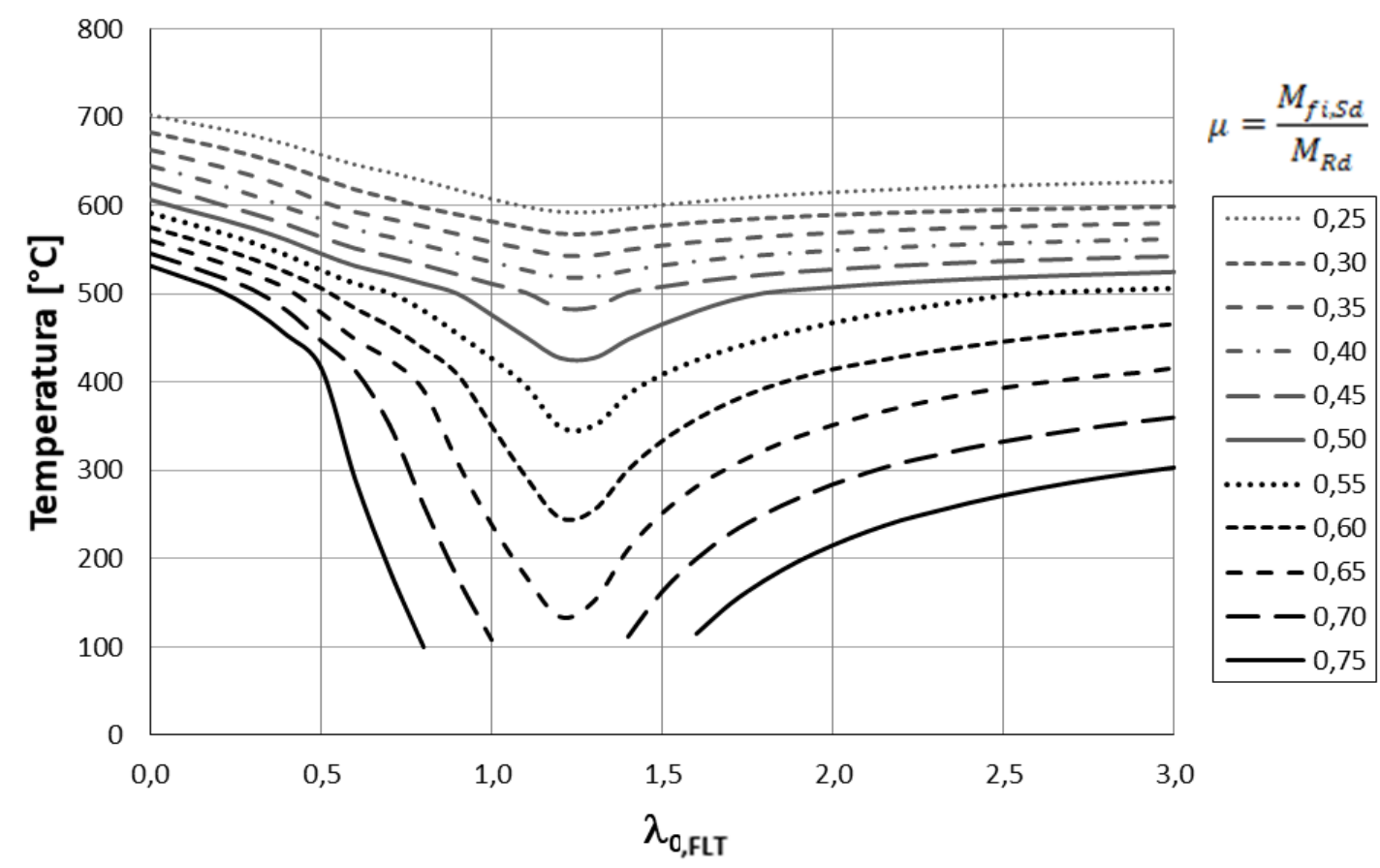

Figura 12 - Temperatura crítica de vigas em função do índice de esbeltez reduzido e do nível de carregamento, para $\mathrm{f}_{\mathrm{y}}=250 \mathrm{MPa}$ e $\mathrm{Z} / \mathrm{W}=1,20$

\section{Temperatura crítica}

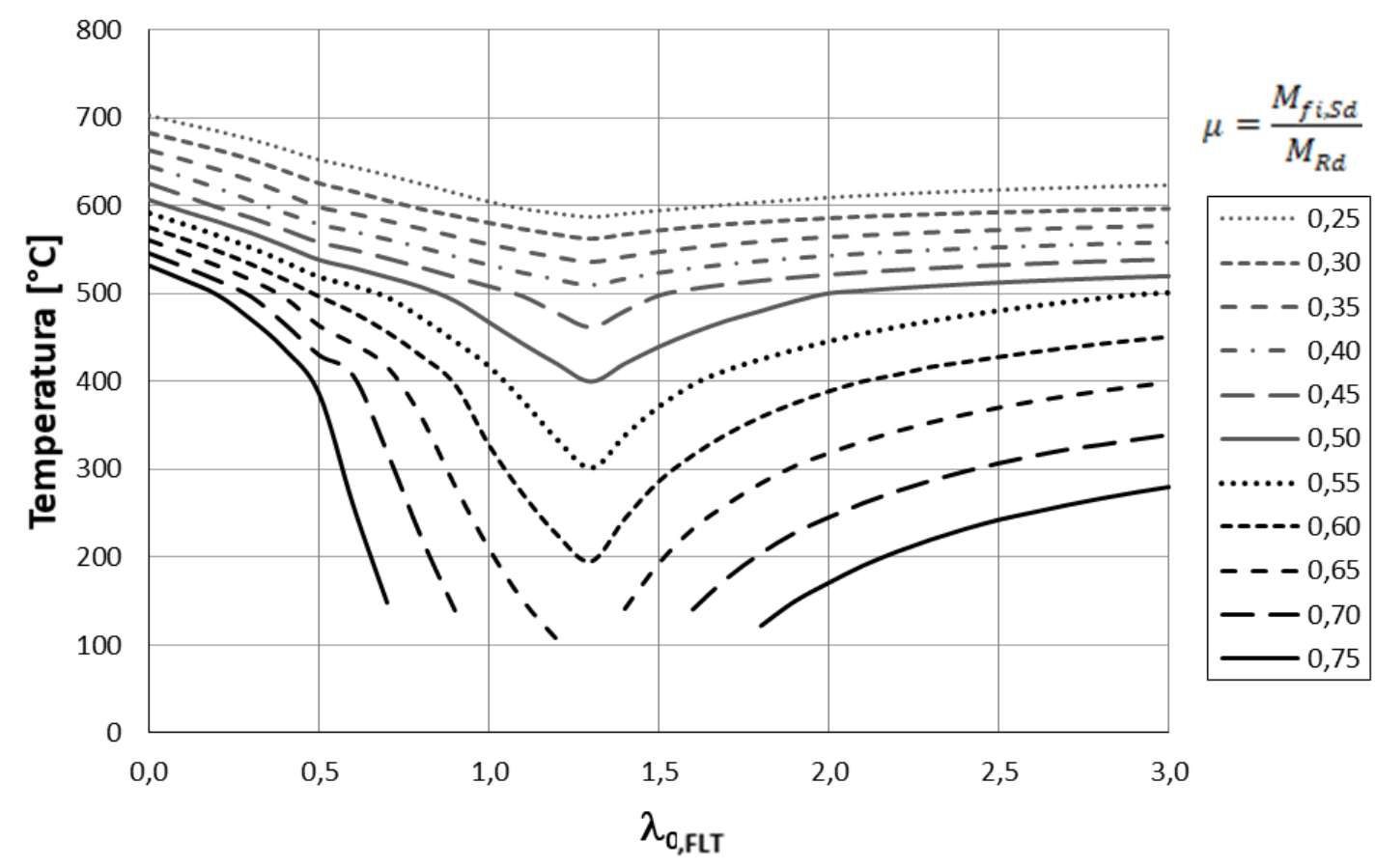


Figura 13 - Temperatura crítica de vigas em função do índice de esbeltez reduzido e do nível de carregamento, para $\mathrm{f}_{\mathrm{y}}=300 \mathrm{MPa}$ e $\mathrm{Z} / \mathrm{W}=1,20$

\section{Temperatura crítica}

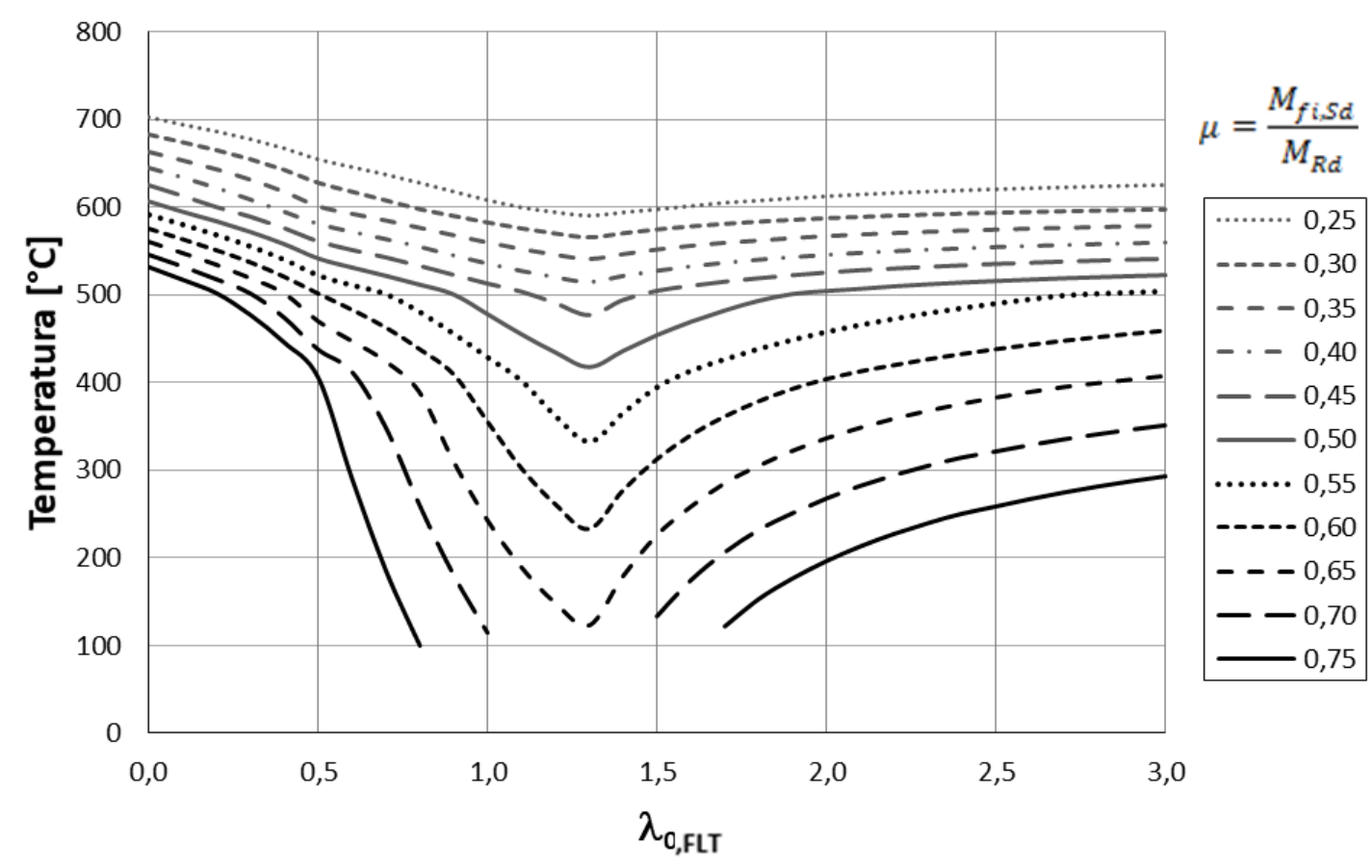

Figura 14 - Temperatura crítica de vigas em função do índice de esbeltez reduzido e do nível de carregamento, para $f_{y}=345 \mathrm{MPa}$ e $\mathrm{Z} / \mathrm{W}=1,20$

\section{Temperatura crítica}

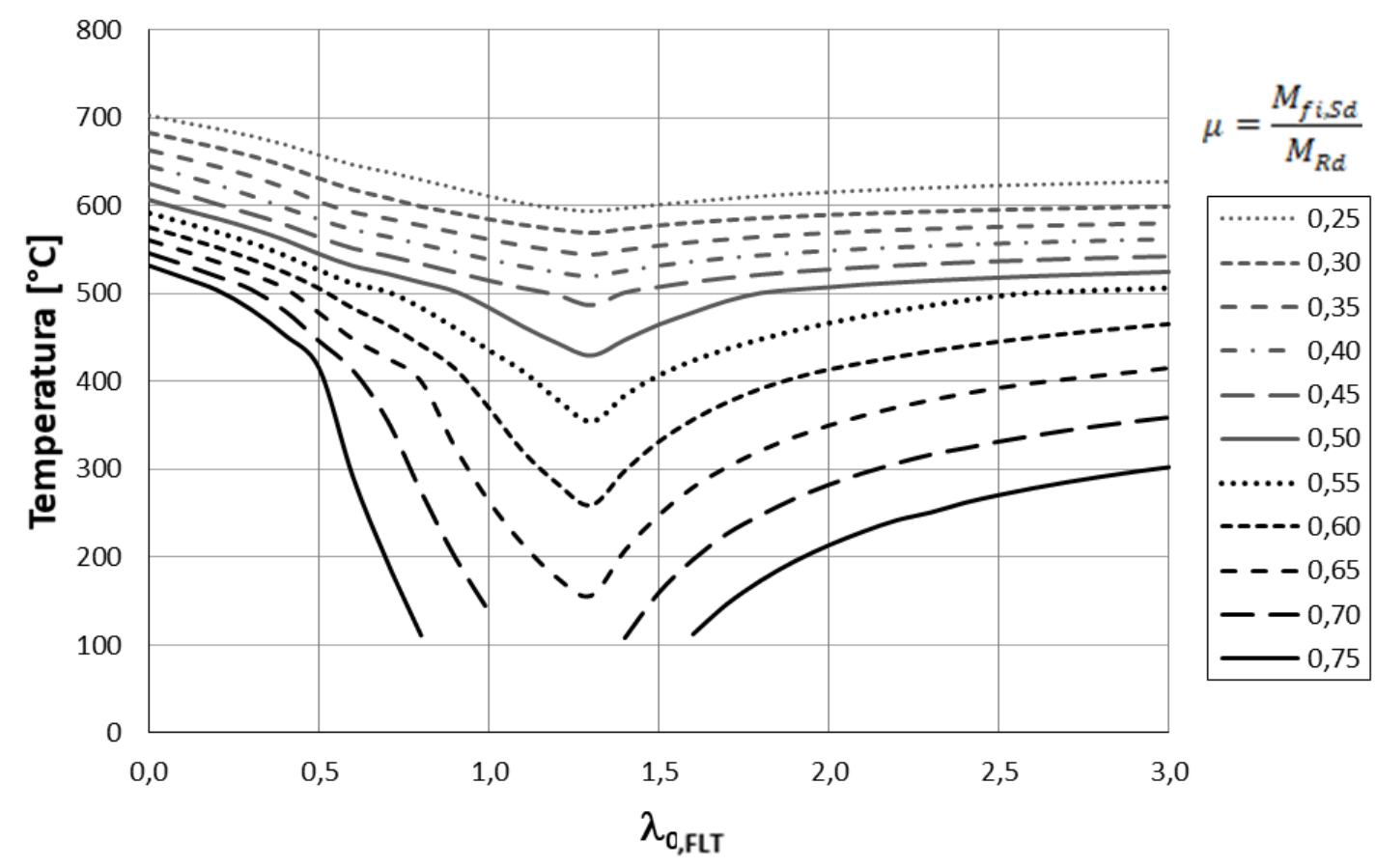


Figura 15 - Temperatura crítica de vigas em função do índice de esbeltez reduzido e do nível de carregamento, para $f_{y}=350 \mathrm{MPa}$ e $\mathrm{Z} / \mathrm{W}=1,20$

\section{Temperatura crítica}

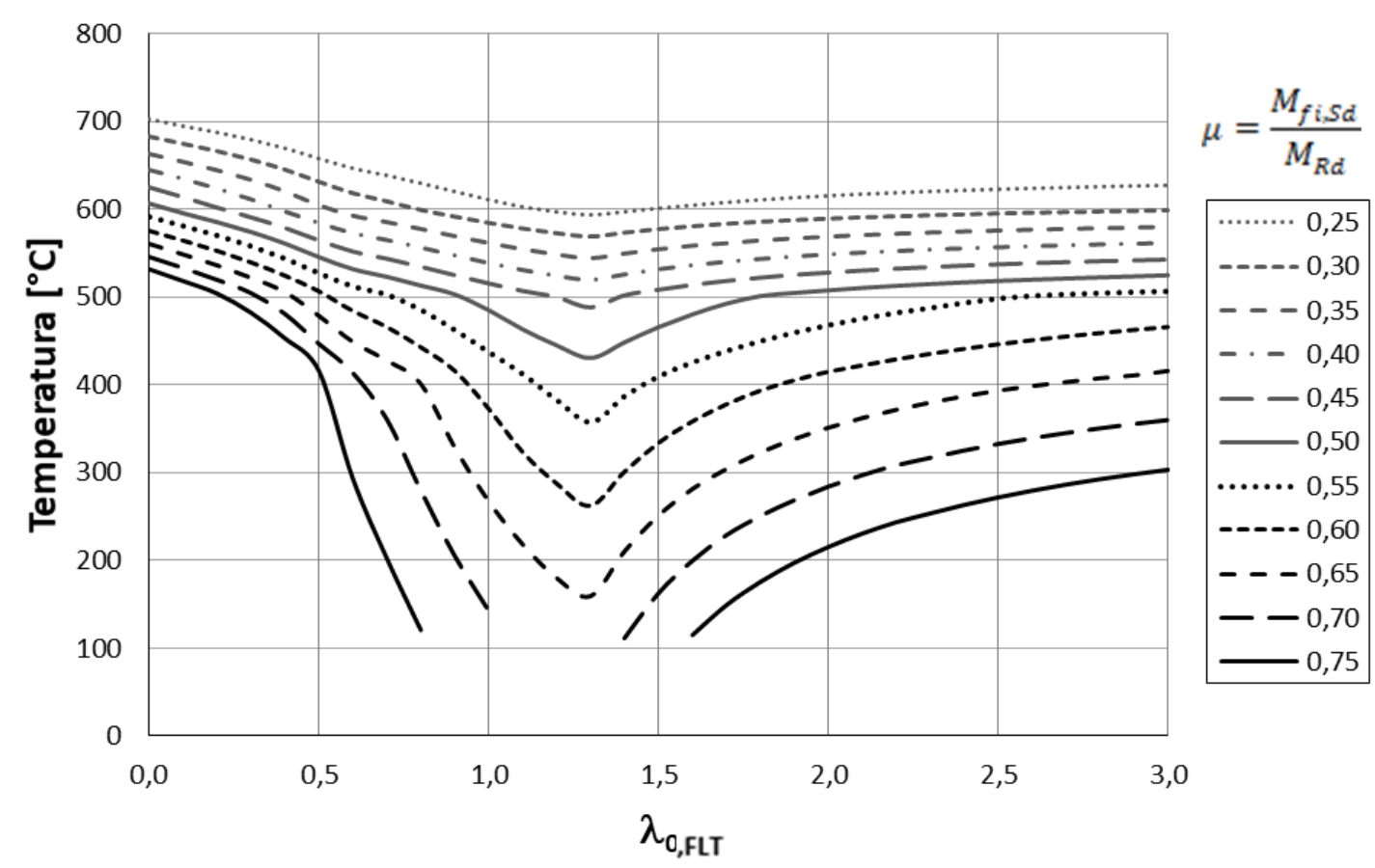

Figura 16 - Temperatura crítica de vigas não mistas sob lajes em função do nível de carregamento

\section{Temperatura crítica}

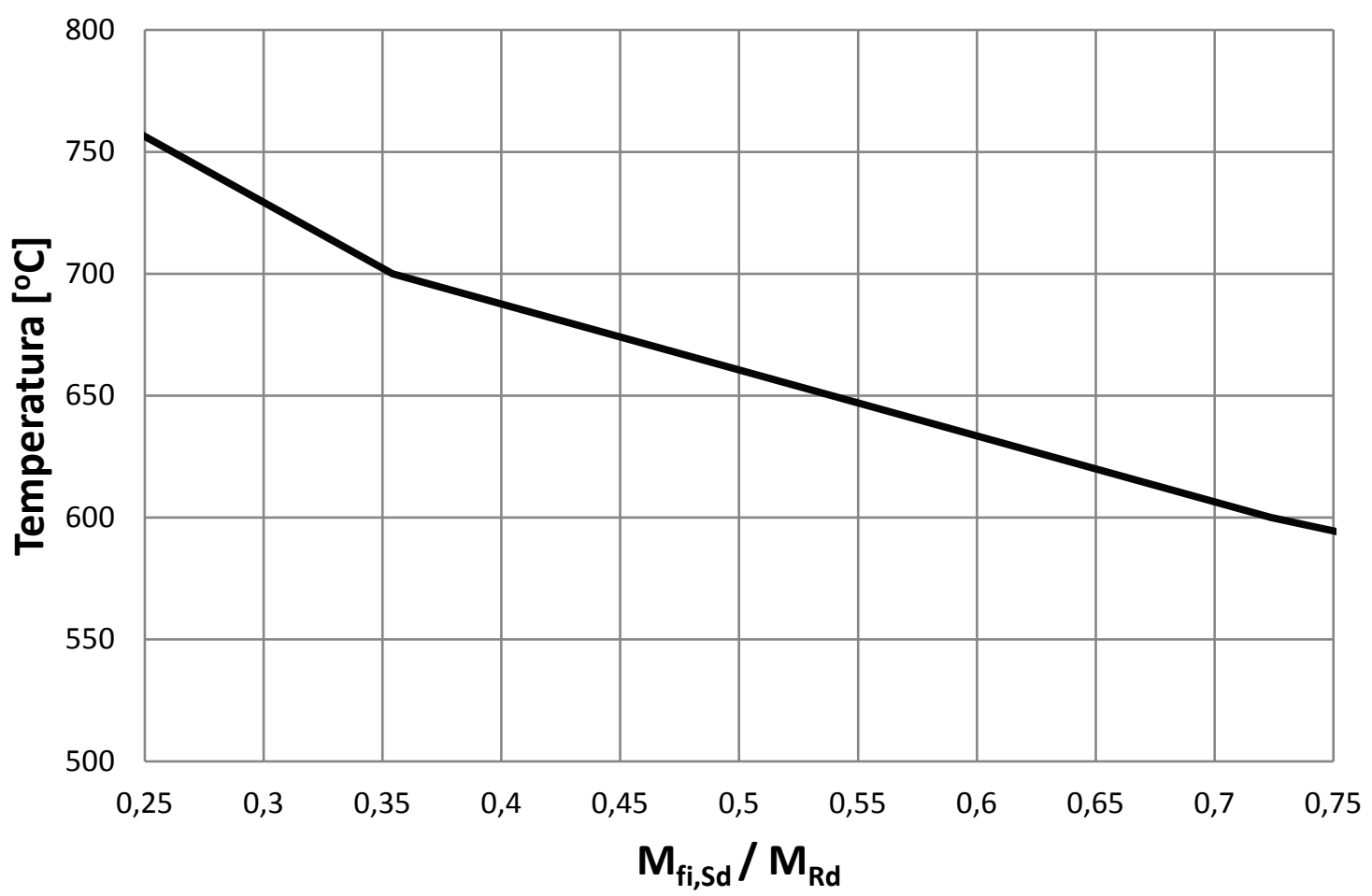


Deve-se ressaltar que os gráficos apresentados são válidos para perfis que não apresentam instabilidade local da alma e da mesa. Além disso, os índices de esbeltez reduzidos em incêndio são aumentados em $15 \%$, o que faz com que perfis que estejam com valores de esbeltez local próximos do limite à temperatura ambiente passem a estar sujeitos à instabilidade local em situação de incêndio. Nos casos da ocorrência de instabilidade local tanto à temperatura ambiente quanto em incêndio, as curvas apresentadas podem ser empregadas para pré-dimensionamento.

Deve-se ter cuidado ao analisar essas curvas, já que à simples vista parece paradoxal que todas as curvas tenham um ponto de mínimo para valores de esbeltez entre 1,0 e 1,5, podendo levar à falsa impressão que a temperatura crítica aumenta se o seu índice de esbeltez reduzido aumentar. Isso se explica lembrando que ao mudar o valor de $\lambda_{0}$ ou

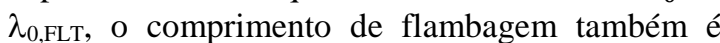
alterado e, por consequência, os valores de $\mathrm{N}_{\mathrm{Rd}} \mathrm{e} \eta$ para pilares e $M_{R d}$ e $\mu$, para vigas, ou seja, para analisar o comportamento da barra deve-se usar outra curva e isso conduzirá a resultados coerentes com o esperado.

As partes de baixo de algumas linhas são interrompidas ao alcançar $100{ }^{\circ} \mathrm{C}$. Nessa temperatura os valores de $\mathrm{k}_{\mathrm{y}, \theta}$ e $\mathrm{k}_{\mathrm{E}, \theta}$ tornam-se unitários, ou seja, não há redução das propriedades do aço.

Nas Figuras 8 a 15, apresentaram-se gráficos adequados a vigas sem travamento lateral. No caso de vigas não mistas, continuamente travadas lateralmente por meio de lajes, a resolução se simplifica pela ausência da instabilidade lateral com torção e tem-se o gráfico mostrado na Figura 16 , válida para qualquer viga I sem instabilidade lateral e local.

Para a construção da Figura 16, foi considerado, também, que a temperatura média é reduzida em função da presença da laje, conforme procedimento recomendado pela NBR 14323 (ABNT, 2013), ou seja, o momento fletor resistente de cálculo considerando o gradiente térmico ao longo da altura da viga vale 1,4 vez o momento fletor resistente de cálculo calculado para a temperatura uniforme.

Na Figura 16, também não foi levada em conta eventual instabilidade local de mesa ou alma.

Na Figura 16, nota-se que entre $600{ }^{\circ} \mathrm{C}$ e $700{ }^{\circ} \mathrm{C}$ a relação é linear e se pode deduzir a Equação 22.

$\theta_{\mathrm{cr}}=270\left(2,94-\frac{\mathrm{M}_{\mathrm{fi}}, \mathrm{Sd}}{\mathrm{M}_{\mathrm{Rd}}}\right)$

Eq. 22

\section{Ações indiretas}

Para o uso das ferramentas apresentadas, o usuário deve conhecer o esforço solicitante de cálculo em situação de incêndio.

As forças e os momentos fletores nodais em situação de incêndio são determinados por análise não linear da estrutura, da mesma forma que se faz à temperatura ambiente, contendo até algumas simplificações, conforme NBR 14323 (ABNT, 2013). Uma dúvida seria a consideração das ações indiretas, ou seja, os efeitos das restrições às deformações térmicas nas extremidades das barras.

No caso de pilares, há aumento da força axial devido à restrição à dilatação, podendo levar à instabilidade (DORR, 2010), no entanto, embora haja necessidade de mais pesquisa, geralmente em procedimentos simplificados tais efeitos são desprezados. Convém ressaltar que os efeitos desse fenômeno não são tão preocupantes em vista de:

(a) a posição deformada decorrente da instabilidade não é estável, ou seja, diferentemente do que ocorre à temperatura ambiente, em que a deformada é estável, a deformada em incêndio é de equilíbrio instável e a barra procura voltar à posição inicial, o que é favorável à estabilidade do pilar (FRANSSEN, 2000; WANG, 2002; BUBACH, 2016);

(b) a deformação axial do pilar e consequente força reativa de restrição são crescentes até certo ponto, quando passam a diminuir devido à redução do módulo de elasticidade (NEVES, 1995; CORREIA, 2011), conforme Figura 17; e

(c) simultaneamente à restrição axial, geralmente ocorre restrição rotacional, o que é favorável à segurança do pilar (CORREIA, 2011; BUBACH, 2016).

Da mesma forma que para os pilares, as restrições vinculares em incêndio de vigas isostáticas não travadas lateralmente, e não são tão preocupantes, pois:

(a) com o aumento de temperatura, a dilatação, para baixas temperaturas, causa reação de compressão, mas com o aumento da temperatura, há um aumento significativo da flecha invertendo a reação para tração e, portanto, favorável à segurança da viga (YIN; WANG, 2004; MOURÃO; SILVA, 2007);

(b) similar ao pilar, a posição deformada decorrente da instabilidade lateral com torção não é estável, ou seja, diferentemente do que ocorre à temperatura ambiente, em que a deformada é estável, a deformada em incêndio é de equilíbrio instável e a barra procura voltar à posição inicial, o que é favorável à estabilidade da viga; e 
simultaneamente à restrição axial, a depender da ligação, pode ocorrer restrição rotacional, o que é favorável à segurança da viga.

Em métodos simplificados (elemento isolado), tanto a NBR 14323 (ABNT, 2013) quanto o Eurocode 3 (EUROPEAN..., 2005) assumem que no caso de ser adotada a exposição ao incêndiopadrão no dimensionamento das barras, os efeitos das expansões térmicas das barras podem ser sempre desprezados, o que facilita bastante a determinação dos esforços solicitantes em incêndio.

Desconsiderando-se tais restrições, o valor de cálculo da ação em situação de incêndio e os correspondentes esforços solicitantes podem ser determinados facilmente seguindo as recomendações da NBR 8681 (ABNT, 2003) ou NBR 14323 (ABNT, 2013) para combinação de ações excepcionais e se pode constatar que são menores do que os correspondentes à temperatura ambiente, por razões estatísticas, isto é, a probabilidade de ocorrência de incêndio quando a ação gravitacional ou eólica produzem as máximas forças sobre a estrutura é diminuta. As normas nacionais (ABNT, 2003, 2013) e estrangeiras (EUROPEAN..., 2001; AMERICAN..., 2010) reconhecem essa baixa probabilidade e permitem reduzir os esforços solicitantes de cálculo em situação de incêndio em relação ao empregado à temperatura ambiente.

\section{Conclusões}

Em métodos simplificados para o dimensionamento de vigas e pilares, as normas brasileira e europeia não consideram as eventuais restrições às deformações térmicas nas extremidades dos perfis. Por meio de análises avançadas tais restrições podem ser simuladas. Embora mais estudos devam ser desenvolvidos, os encontrados até o momento levam a crer que os métodos normatizados, que desprezam os efeitos das restrições, conduzem a bons resultados, além do que tornam desnecessário o uso de análises térmicas computacionais, o que facilita sobremaneira o meio técnico.

Os autores deste artigo desenvolveram o programa AcoInc, que inclui várias utilidades para o estudo de perfis I em situação de incêndio. Uma delas é a determinação da temperatura crítica de vigas e pilares.

Foram apresentados neste trabalho gráficos que permitem determinar de forma expedita a temperatura crítica de vigas e pilares, não sujeitos a instabilidades locais, em função do índice de esbeltez reduzido à temperatura ambiente e do nível de carregamento do elemento em situação de incêndio.

Não foi encontrado na literatura pesquisada nada que se assemelhe ao aqui apresentado.

$\mathrm{O}$ valor da temperatura crítica igual a $550{ }^{\circ} \mathrm{C}$, bastante empregado na prática, pode ser muito diferente, a depender da situação estrutural.

Figura 17 - Desenvolvimento das forças de restrição

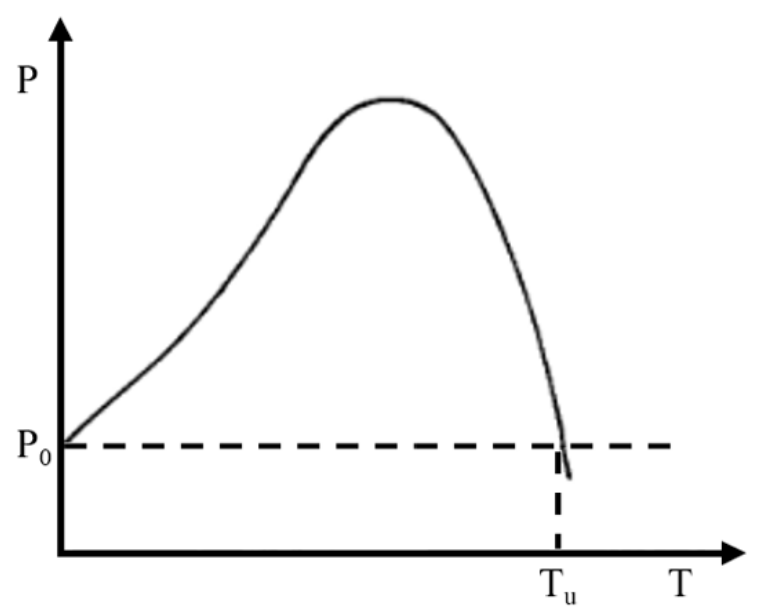

Fonte: Neves (1995 apud BUBACH, 2016).

${ }^{1}$ NEVES, I. C. The Critical Temperature of Steel Columns With Restrained Thermal Elongation. Fire Safety J ournal, v. 24, p. 211-227, 1995. 
O dimensionamento em situação de incêndio, em que se compara a temperatura crítica à temperatura atuante nos perfis, base dos métodos normatizados, simplifica-se muito caso se empreguem os gráficos aqui apresentados. Entretanto, cabe ressaltar que podem ser menos econômicos se comparados a resultados de análises de subconjuntos estruturais (por exemplo: vigas mistas sob lajes mistas ou pilares junto a paredes) que levam a campos de temperatura não uniformes. Porém, demandariam análises computacionais ou experimentais complexas. Ainda assim, as temperaturas críticas encontradas por intermédio dos gráficos podem servir como pré-dimensionamento.

Para trabalhos futuros, mantendo o objetivo de criar ferramentas simples para uso do meio técnico não especializado em incêndio, pretende-se estudar estruturas mais complexas e incluir os efeitos das instabilidades locais.

\section{Referências}

AMERICAN INSTITUTE OF STEEL CONSTRUCTION. ANSI/AISC 360: specification for structural steel buildings. Chicago, 2010.

\section{ASSOCIAÇÃO BRASILEIRA DE NORMAS}

TÉCNICAS. NBR 14323: dimensionamento de estruturas de aço de edifícios em situação de incêndio. Rio de Janeiro, 2013.

ASSOCIAÇÃO BRASILEIRA DE NORMAS TÉCNICAS. NBR 14323: dimensionamento de estruturas de aço de edifícios em situação de incêndio. Rio de Janeiro, 1999.

ASSOCIAÇÃO BRASILEIRA DE NORMAS TÉCNICAS. NBR 8681: ações e segurança nas estruturas. Rio de Janeiro, 2003

ASSOCIAÇÃO BRASILEIRA DE NORMAS TÉCNICAS. NBR 8800: projeto de estruturas de aço e de estruturas mistas de aço e concreto de edifícios. Rio de Janeiro, 2008.

ASSOCIAÇÃO BRASILEIRA DE NORMAS TÉCNICAS. NBR 8800: projeto e execução de estruturas de aço de edifícios. Rio de Janeiro, 1988.

BUBACH, C. R. Pilares de Aço Com Restrição Axial e Rotacional em Situação de Incêndio. Vitória, 2016. Dissertação (Mestrado em Engenharia Civil) - Escola de Engenharia Civil, Universidade Federal do Espírito Santo, Vitória, 2016.
CORREIA, A. J. P. M. Fire Resistance of Steel and Composite Steel-Concrete Columns. Coimbra, 2011. Tese (Doutorado em Engenharia Civil) - Faculdade de Ciências e Tecnologia, Universidade de Coimbra, Coimbra, 2011.

DORR, J. B. Modelos Numéricos de Pilares de Aço em Situação de Incêndio Considerando a Influência da Restrição Axial. São Carlos, 2010. Dissertação (Mestrado em Engenharia Civil) Escola de Engenharia, Universidade de São Paulo, São Carlos, 2010.

\section{EUROPEAN COMMITTEE FOR}

STANDARDIZATION. EN 1990 Eurocode 0:

Basis of structural design. Brussels, 2001.

\section{EUROPEAN COMMITTEE FOR}

STANDARDIZATION. EN 1993 Eurocode 3:

design of steel structures: part 1-2: general rules: structural fire design. Brussels, 2005.

FRANSSEN, J. M. Failure Temperature of a System Comprising a Restrained Column Submitted to Fire. Fire Safety Journal, v. 34, p. 191-207, 2000.

FRANSSEN, J. M.; ZAHARIA, R. Design of Steel Structures subject to Fire. Liège: Université de Liège, 2005.

FRANSSEN, J. M.; ZAHARIA, R.; KODUR, V. Designing Steel Structures for Fire Safety. CRC Press, 2009.

GARLOCK, M.; KODUR, V. (Eds.). Proceedigns of the 9th International Conference on Structures in Fire SiF'16. Princeton, 2016.

MELÃO. A. R. Sobre Perfis I de Aço em Situação de Incêndio Paramétrico. São Paulo, 2016. Dissertação (Mestrado em Engenharia Civil) - Escola Politécnica, Universidade de São Paulo, São Paulo, 2016

MOURÃO, H. R.; SILVA, V. P. On the Behaviour of Single-Span Steel Beams Under Uniform Heating. Journal of the Brazilian Society of Mechanical Sciences and Engineering, v. 29, p. 115-122, 2007

PARKINSON, D.; KODUR, V. PerformanceBased of Structural Steel for Fire Conditions. Amer Society of Civil Engineers, 2008.

SILVA, V. P. Estruturas de Aço em Situação de Incêndio. São Paulo: Zigurate, 2004.

SILVA, V. P. Estruturas Metálicas e de Madeira. Dimensionamento de Estruturas de Aço. Notas de aula. São Paulo. 2012.

SILVA, V. P. Sobre a Instabilidade de Barras Comprimidas. Revista da Estrutura de Aço, v. 5, p. 79-98, 2016. 
SKOWRONSKI, W. Fire Safety of Metal Structures. Theory and Design Criteria. Varsóvia: Polish Scientific Publishers, 2004.

VELARDE, J. S. S.; SILVA, V. P. Sobre o Dimensionamento de Pilares de Aço em Situação de Incêndio. Revista Sul-Americana de Engenharia Estrutural, Passo Fundo, v. 6, n. 2/3, 2009.

VILA REAL, P. Incêndio em Estruturas Metálicas: cálculo estrutural. Orion, 2003.

VILA REAL, P.; FRANSSEN, J. M. Fire Design of Steel Structures. European Convetion for Constructional Steelwork.Wiley, 2010.

WANG, Y. C. Steel and Composite Structures Behaviour and Design for Fire Safety. New York: Spon Press, 2002.
WANG, Y. et al. Performance-Based Fire Engineering of Structures. New York: Span Press, 2013.

YIN, Y. Z.; WANG, Y. C. A Numerical Study of Large Deflection Behaviour of Restrained Steel Beams at Elevated Temperatures. Journal of Constructional Steel Research, v. 60, n. 7, 2004.

\section{Agradecimentos}

Os autores agradecem à Fundação de Amparo à Pesquisa do Estado de São Paulo (Fapesp), ao Conselho Nacional de Desenvolvimento Científico e Tecnológico (CNPq) e à Coordenação de Aperfeiçoamento de Pessoal de Nível (Capes).

\section{Valdir Pignatta Silva}

Departamento de Engenharia de Estruturas e Geotecnia, Escola Politécnica | Universidade de São Paulo | Av. Professor Luciano Gualberto, Trav. 3, 380, Edifício da Engenharia Civil, Butantã | São Paulo - SP - Brasil | CEP 05508-900 | Tel.: (11) 3091-5562 | E-mail: valpigss@usp.br

\section{Arthur Ribeiro Melão}

Departamento de Engenharia de Estruturas e Geotecnia, Escola Politécnica | Universidade de São Paulo | E-mail: melao@usp.br

Revista Ambiente Construído

Associação Nacional de Tecnologia do Ambiente Construído

Av. Osvaldo Aranha, 99 - 3o andar, Centro

Porto Alegre - RS - Brasil

CEP $90035-190$

Telefone: +55 (51) 3308-4084

Fax: +55 (51) 3308-4054

www. seer. ufrgs. br/ ambienteconstruido

E-mail: ambienteconstruido@ufrgs.br

(c) () This is an open-access article distributed under the terms of the Creative Commons Attribution License. 